\title{
Clustering properties of produced particles in high-energy $p p$ collisions
}

\author{
Cheuk-Yin Wong $\odot,{ }^{1}$ Hanpu Jiang, ${ }^{2}$ Nanxi Yao, ${ }^{2}$ Liwen Wen, ${ }^{2}$ Gang Wang, ${ }^{2}$ and Huan Zhong Huang ${ }^{2,3}$ \\ ${ }^{1}$ Physics Division, Oak Ridge National Laboratory, Oak Ridge, Tennessee 37831, USA \\ ${ }^{2}$ Department of Physics and Astronomy, University of California, Los Angeles, California 90095, USA \\ ${ }^{3}$ Key Laboratory of Nuclear Physics and Ion-beam Application (MOE) and Institute of Modern Physics, \\ Fudan University, Shanghai 200433, China
}

(Received 2 January 2018; accepted 17 August 2020; published 8 September 2020)

\begin{abstract}
Minijets provide useful information on parton interactions in the low transverse-momentum (low- $p_{T}$ ) region. Because minijets produce clusters, we study the clustering properties of produced particles in highenergy $p p$ collisions as a first step to identify minijets. We develop an algorithm to find clusters by using the k-means clustering method, in conjunction with a k-number (cluster number) selection principle in the space of pseudorapidity and azimuthal angles. We test the clustering algorithm using events generated by PYTHIA8.1 for $p p$ collision at $\sqrt{s}=200 \mathrm{GeV}$. We find that clustering of low- $p_{T}$ hadrons occurs in high multiplicity events. However, similar clustering properties are also present for particles produced randomly in a finite pseudorapidity and azimuthal angle space. To distinguish the dynamics from random generations of events, it is necessary to examine the correlation between particles and between clusters. We find that the correlations between clusters may provide a useful tool to distinguish the underlying dynamics of the reaction mechanism.
\end{abstract}

DOI: 10.1103/PhysRevD.102.054007

\section{INTRODUCTION}

The mechanism of relativistic parton-parton hard scattering is an important basic perturbative QCD particle production process in high-energy nucleon-nucleon collisions [1-29]. Because of the composite nature of a nucleon, multiple hard scattering between partons of the projectile and target nucleons will lead to the production of jets and dijets whose subsequent fragmentation gives rise to the production of particle clusters. It is different from the nonperturbative flux-tube fragmentation process $[6,10,25,30-46]$ in which a quark of one nucleon and the diquark of the other nucleon (or a gluon of one nucleon and the gluon of the other nucleon [47-52]) form one flux tube and the subsequent fragmentation of the flux tube leads to the production of hadrons. It is also different from the direct-fragmentation process [53] in which the partons from the composite nucleon fragment directly into the detected particles.

The hard-scattering process was originally proposed as the dominant process for the production of high- $p_{T}$ jet clusters of order many tens of $\mathrm{GeV} / \mathrm{c}$ [1-7]. However, the UA1 Collaboration found that it is also the dominant process for the production of particle clusters with a total $p_{T}$ of a few

Published by the American Physical Society under the terms of the Creative Commons Attribution 4.0 International license. Further distribution of this work must maintain attribution to the author(s) and the published article's title, journal citation, and DOI. Funded by SCOAP.
GeV/c for $p \bar{p}$ collisions at $\sqrt{s}=0.2$ to $0.9 \mathrm{TeV}$ [15]. The term "minijet" was introduced to describe low- $p_{T}$ jet clusters [16]. The dominance of jet production was found to extend to lower $p_{T}$ domains at high collision energies because (i) the fraction of particles produced by such a process increases rapidly with collision energies $\sqrt{s}$, and (ii) the jet-production invariant cross section at midrapidity varies as an inverse power of $p_{T}[8,16,17,27,29,54]$.

Recently, the region of dominance of the hard-scattering process has been found to extend to the production of hadrons even to the lower $p_{T}$ region of a few tenths of a $\mathrm{GeV} / \mathrm{c}$ [26-29]. An indirect piece of evidence comes from the observation on the transverse momentum spectra of produced hadrons: For the production of particles with $p_{T}$ within the range from a few tenths of a GeV to a few hundred $\mathrm{GeV}$ in high-energy $p p$ and $p \bar{p}$ collisions at $\sqrt{s}=0.9$ to $7 \mathrm{TeV}$, the hadron transverse spectra, whose magnitude spans over 14 decades of magnitude, can be described by a simple Tsallis inverse-power-law type distribution with only 3 degrees of freedom [26-29]. The simplicity of the powerlaw type transverse spectra suggests that only a single mechanism, the hard-scattering process, dominates over the extended $p_{T}$ domain. An additional piece of direct evidence comes from the jetlike structure in the two-hadron angular $(\Delta \eta, \Delta \phi)$ correlation data in a minimum- $p_{T}$-bias measurement of the STAR Collaboration in $p p$ collisions at $\sqrt{s}=200 \mathrm{GeV}$ [55-58]. The momentum distributions of hadrons associated with a hadron trigger of a few $\mathrm{GeV} / \mathrm{c}$ in $p p$ collisions at the same energy exhibit a jetlike cluster 
structure within a cone in a similar manner, as observed by the STAR Collaboration [59-66] and the PHENIX Collaboration [66,67].

The extension of the dominance of the hard-scattering model to the low- $p_{T}$ domain of a few tenths of $\mathrm{GeV} / \mathrm{c}$ raises serious questions on the large and divergent perturbative quantum chromodynamics ( $\mathrm{pQCD}$ ) corrections at low $p_{T}$ and the competition from nonperturbative flux tube fragmentation process associated with low- $p_{T}$ phenomena. We need additional theoretical and experimental comparisons of the hard-scattering model to construct the proper phenomenological description in the low- $p_{T}$ region.

If the hard-scattering process of the $p p$ collision is appropriate also for the low- $p_{T}$ region, then multiple parton interactions (known also as multiple collision processes) [1,7,11-14] must occur to produce multiple minijets and mini-dijets [1,7,8,11-17]. Among many other diagrams, the hard-scattering process can lead to the production of one, two, and three pairs of mini-dijets as depicted in Figs. 1(a)-1(c). Furthermore, a parton of one proton can make multiple collisions (known also as rescattering [14]) with different partons of the other proton, as depicted in Figs. 1(d)-1(f). The numbers of produced minijets can be even, as in Figs. 1(a)-1(c), or odd, as in Figs. 1(d)-1(f). There can also be additional higher-order diagrams with the radiation and the absorption of gluon partons, which lead to additional minijets.

The multiple parton scattering processes in the production of high- $p_{T}$ jets have been observed in high-energy $p p$ or $p \bar{p}$ collisions [68-71]. Theoretical discussions on the production of minijets beyond the leading order has been investigated, and hard inclusive dijet production with multiparton interactions has also been considered [18-24,27,72]. However, in the low- $p_{T}$ region, the experimental investigation for

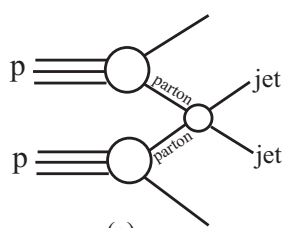

(a)

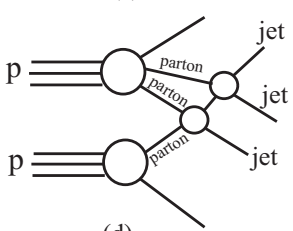

(d)

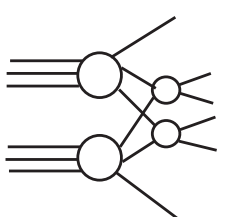

(b)

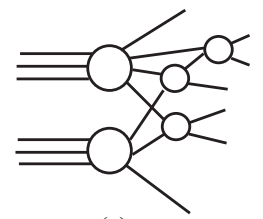

(e)

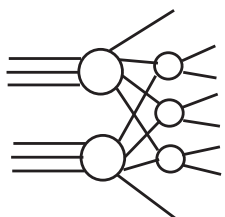

(c)

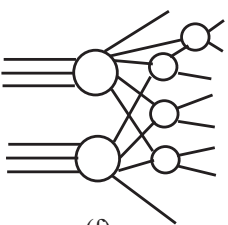

(f)
FIG. 1. Various multiple collision diagrams in a $p p$ hard scattering leading to the production of jets, which are called minijets when the transverse momentum of the jet is small. Shown here are diagrams for the production of (a) a dijet pair, (b) two dijet pairs, and (c) three dijet pairs. Furthermore, a scattered parton can make an additional collision with a different parton of the other proton, as shown in diagrams (d), (e), and (f). multiple parton interactions with the production of multiple minijets and mini-dijets remains lacking.

We would like to develop tools to study multiple hardscattering processes for the production of multiple minijets and mini-dijets in the low- $p_{T}$ domain in $p p$ collisions at high energies. As a first step, we examine here the clustering properties of minijets in the pseudorapidity and azimuthal angle space and search for an algorithm to assist the finding of minijet clusters candidates.

The minijet processes in a nucleon-nucleon collision are not only intrinsically important with regard to our understanding of the underlying mechanism for low- $p_{T}$ particle production, they are also extrinsically valuable in applications because nucleon-nucleon collisions lie at the heart of a nucleus-nucleus collision, and the low- $p_{T}$ particle production dominates the particle production process. An understanding of the mechanism of low- $p_{T}$ particle production in nucleon-nucleon collisions provide vital information on the initial condition that may exist at the early stage of nucleusnucleus collisions, on which much interest has been focused recently. In particular, the observation of the near-side jet and the away-side ridge in high-multiplicity events in highenergy $p p$ collisions [59-66,73-80] indicates that the initial dynamics of the system after the production of a jet or a minijet [79] depends on the initial configuration of the system. The examination of such a system also calls for an event-by-event study of the multiple minijet and mini-dijet productions in $p p$ collisions.

Our event-by-event study has been stimulated by a similar investigation for particle production at lower $p p$ collision energies, where the particle production process may be dominated by flux-tube fragmentation [81]. There, the basic conservation laws and the semiclassical picture of the fragmentation process provide powerful tools to reconstruct the space-time dynamics of the pair production processes that may occur, if exclusive data for the production process are available. In the present investigation, the space-time dynamics of parton-parton hard scattering may provide useful experimental information on the multiple collision processes and on the constituent nature of the colliding nucleons.

In the search for separated minijet and mini-dijets, one of the important ingredients is the $p_{T}$ threshold value that sets the $p_{T}$ limit for the inclusion of a particle as part of a minijet. Clearly, the higher the $p_{T}$ limit, the cleaner will be the cluster and their possible corresponding minijet partners. On the other hand, the higher the $p_{T}$ value, the lower will be the number of cluster counts and the lower the sampling statistics. Furthermore, because each minijet occupies a substantial area in $(\eta, \phi)$ space, the limited angular and azimuthal space may make the separation of the minijets a more difficult task. In the present manuscript, we shall use the minimum-bias selection of particles with $p_{T} \geq 0.15 \mathrm{GeV} / \mathrm{c}$. An optimum $p_{T}$ limit and cluster multiplicity will need to be searched for in realistic applications with real data. 
This paper is organized as follows. In Sec. II, we summarize the properties of a minijet from previous studies. In Sec. III, we exhibit the distribution of produced charged hadrons in the whole range of rapidity and azimuthal angles for sample minimum-biased PYTHIA calculations to illustrate the occurrence of clusters for $p p$ collisions at $\sqrt{s_{p p}}=200 \mathrm{GeV}$. In Sec. IV, we introduce the algorithm for finding clusters in the pseudorapidity and azimuthal angle space. The algorithm consists of the k-means clustering method supplemented by the k-number (cluster number) selection principle, based on the physical properties of minijet clusters. We illustrate the usage of such an algorithm in Sec. V, using sample events with high multiplicities generated by PYTHIA8.1. We examine the change of the clustering behavior as a function of increasing multiplicities in PYTHIA8.1 events in Sec. VI. We investigate whether similar properties of clustering can be found in a random distribution within the same finite $(\eta, \phi)$ phase space in Sec. VII. We study the correlation between particles and between clusters in Sec. VIII. We present our conclusions and discussions in Sec. IX. We discuss another method of finding the cluster number, the elbow method, and note its ambiguities in the Appendix. For completeness, we also include the results of the azimuthal angular correlations and pseudorapidity correlations in the Appendix.

\section{PROPERTIES OF A MINIJET}

The structure of a minijet in the $(\eta, \phi)$ scatter plot can be inferred from the distribution of the two-hadron angular correlation as a function of the pseudorapidity difference $\Delta \eta=\eta_{2}-\eta_{1}$ and the azimuthal angular differences $\Delta \phi=$ $\phi_{2}-\phi_{1}$ of the two particles detected with angular coordinates $\left(\eta_{1}, \phi_{1}\right)$ and $\left(\eta_{2}, \phi_{2}\right)$ in coincidence [55-67]. For $p p$ collisions at $\sqrt{s}=200 \mathrm{GeV}$, the minijet structure appears as a cluster of particles in the $(\eta, \phi)$ space (and a cone in three-dimensional configuration space) as indicated by a two-hadron Gaussian distribution in $\Delta \eta$ and $\Delta \phi$ in the form

$$
\frac{d N}{d \Delta \eta d \Delta \phi}(\Delta \eta, \Delta \phi) \propto \exp \left\{-\frac{\left.(\Delta \eta)^{2}+\Delta \phi\right)^{2}}{2 \sigma_{\phi}^{2}}\right\},
$$

where the quantity $\sigma_{\phi}$ was found to be [66]

$\sigma_{\phi}=\frac{\sigma_{\phi 0} m_{a}}{\sqrt{m_{a}^{2}+p_{T, \text { trigger }}^{2}}}, \quad \sigma_{\phi 0}=0.5, \quad m_{a}=1.1 \mathrm{GeV}$,

when triggered by a hadron with transverse momentum $p_{T, \text { trigger. }}$. It should, however, be emphasized that the Gaussian form of the distribution in Eq. (1) is only a hypothesis. Actual shape of the distribution will require the identification and the knowledge of minijets and all their individual member particles, which are not yet generally available. In the minimum-bias data at the Relativistic Heavy Ion Collider energies, we shall consider the quantity $p_{T \text {,trigger }}$ takes on the value of $\sqrt{\left\langle p_{T}^{2}\right\rangle}$, which is of order $0.4 \mathrm{GeV} / \mathrm{c}$. Equation (2), therefore, yields $\sigma_{\phi} \simeq 0.5$. The two-particle distribution of Eq. (1) has a half width at half maximum at $R=\sqrt{(\Delta \eta)^{2}+(\Delta \phi)^{2}}=1.2 \sigma_{\phi}=0.6$. We can consider a circle of radius $R$ in the $(\eta, \phi)$ plane. The minimum separation between any two points inside the circle is zero and the maximum separation is $2 R$. Setting $2 R=2.4 \sigma_{\phi}$ (or $R=0.6$ ) will allow the circle to contain a large fraction (about 95\%) of the Gaussian distribution (1) within the circular domain. It is reasonable to assume that a signature of a minijet cluster of particles is indicated by a cluster of particles within a radius of $R \simeq 0.6$ in the plane of $(\eta, \phi)$.

In the hard-scattering process in the collision of two partons, $a+b \rightarrow a^{\prime}+b^{\prime}$, the partons $a^{\prime}$ and $b^{\prime}$ materialize subsequently as minijets. The initial $a$ and $b$ partons may be endowed with a small intrinsic transverse momentum $k_{T}$ of the order of 0.6 to $1.0 \mathrm{GeV} / \mathrm{c}[3,82-84]$. The conservation of 4-momentum requires that the scattered partons $a^{\prime}$ and $b^{\prime}$ will come out azimuthally in nearly back-to-back directions. The signature of a mini-dijet can be taken to be a pair of minijets whose azimuthal angles are approximately correlated within the range of $\pi-R$ to $\pi+R$.

\section{DISTRIBUTIONS OF PRODUCED HADRONS IN SAMPLE PYTHIA EVENTS}

The description, in terms of partons, is useful only in the early stages of the $p p$ collision. Subsequent evolution of the partons will require their hadronization into detectable hadrons. The dynamics of particle production processes leaves an imprint on the distribution of the produced particles.

Our knowledge of how partons hadronize remains incomplete. We wish to obtain some insight on the dynamics of the hadronization processes by examining the distribution of the produced particles on an event-byevent basis. To see what may be expected, it is instructive to study the distribution of produced particles in the PYTHIA6.4 calculations with its hadronization model in which the history of the evolution of the partons are recorded and traceable [6].

In the PYTHIA6.4 description of the $p p$ collision [6], valence quarks, valence diquarks, and gluon partons are produced, and they can be arranged into two initial strings connected by leading valence quarks and antiquarks (or diquarks). The produced gluons are then split into quarkantiquark pairs and the quarks and their neighboring antiquark (or diquark) are connected into segments of shorter "kinky substrings". Each substring is subsequently fragmented to produce quark-antiquark pairs in accordance with the nonperturbative Lund string fragmentation model. In the Lund model, the fragmentation of the substring segments follows the outside-inside cascades by producing 
a quark-antiquark pair carrying a light-cone momentum fraction in accordance with a given fragmentation function. The $q-\bar{q}$ pair production leads to a shorter remainder string with a smaller invariance mass, and the end parton particles continue to repeat the string fragmentation process until the invariance mass of the remainder string becomes lower than the limit. After the fragmentation of the kinky substrings, neighboring $q$ and $\bar{q}$ (or diquark) are then connected to form hadrons. The production of the $q-\bar{q}$ pairs leads to clusters of hadrons that are likely to be correlated at the end points and along the string. The outside-inside cascade of string fragmentation of the leading partons of the string in the Lund model is mathematically and kinematically similar to the parton cascade in high- $p_{T}$ leading parton fragmentation and parton showering, differing mainly in the nature of the fragmentation functions.

In a PYTHIA event, charged and neutral hadrons, as well as photons are produced. We shall focus on minimumbiased events without any $p_{T}$ selection. They reside within the window of $y_{\text {target }} \leq \eta \leq y_{\text {beam }}$, and $-\pi \leq \phi \leq \pi$ in the $(\eta, \phi)$ plane, where $y_{\text {beam }}=-y_{\text {target }}=5.29$ for $p p$ collisions at $\sqrt{s}=200 \mathrm{GeV}$.

The scatter plots of produced charged hadrons obtained in PYTHIA6.4 for a few randomly selected sample events,
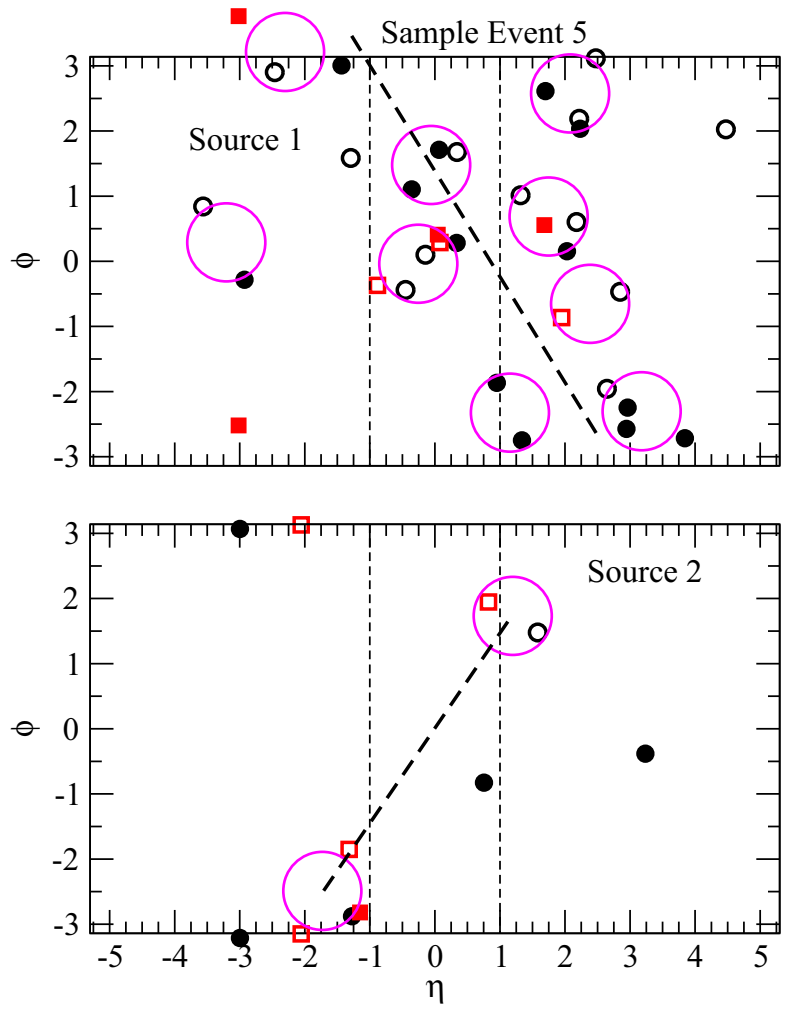

FIG. 2. Scatter plots in the $(\eta, \phi)$ plane for produced charged particles in the full $(\eta, \phi)$ plane in a randomly selected sample event 5 generated by PYTHIA6.4 for $p p$ collisions at $\sqrt{s}=200 \mathrm{GeV}$. Cluster circles with a radius $R=0.6$ are plotted to circumscribe the cluster centers. Some of the data points are wrapped around to facilitate cluster association. event 5 and event 6, are displayed in Figs. 2 and 3. Each of the $p p$ events contains two separate quark-diquark sources of strings or partons. A string source will produce particles by string fragmentation, whereas a parton source will collide to produce particles by the hard-scattering processes. To gain some insight on the $p_{T}$ and the charge of the produced particles, we use circular and square points to indicate $p_{T}$ less and greater than $0.5 \mathrm{GeV} / \mathrm{c}$, respectively, with solid points for positive particles and open points for negative particles. In each event, the intermediate outputs from PYTHIA6.4 allow the specification of the two separate strings or partons from which the produced charged hadrons originate. The patterns of hadron particles reveal many interesting characteristics. One observes that produced particles tend to form clusters. A circle of radius $R=0.6$ and a minimum of two hadrons can be conveniently used to separate different clusters, as such, a definition leaves very few numbers of hadrons outside the clusters. For each string, the clusters appear correlated to form roughly a linear pattern along the axis indicated by the dashed lines in Figs. 2 and 3. Because of the fragmentation of kinky substrings along the greater parent string, clusters have a tendency to correlate with an azimuthal angular difference of about $\pi$. Source 1 of event
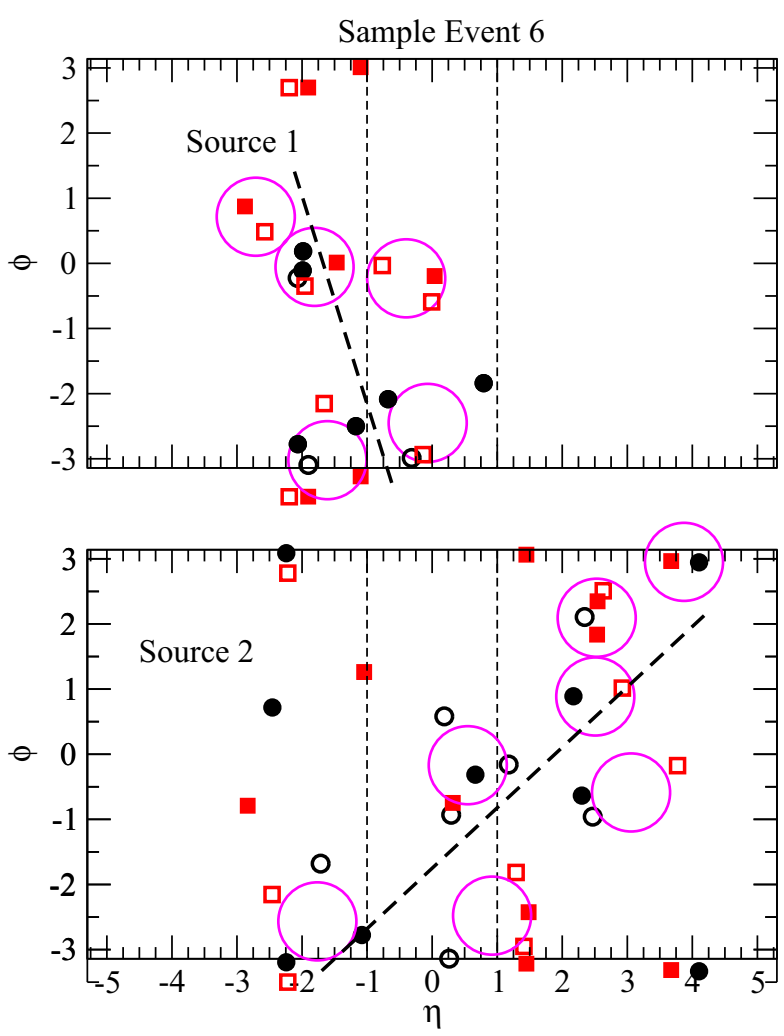

FIG. 3. Scatter plots in the $(\eta, \phi)$ plane for produced charged particles in the full $(\eta, \phi)$ plane in sample events generated by PYTHIA6.4 for $p p$ collisions at $\sqrt{s}=200 \mathrm{GeV}$. Cluster circles with a radius $R=0.6$ are plotted to circumscribe the cluster centers. 
5 in Fig. 2 and source 2 of event 6 in Fig. 3 give a large number of clusters along the parent string. They appear to bear the characteristic of a string fragmentation. Source 2 of event 5 and source 1 of event 6 gives two groups of clusters, which are roughly back-to-back correlated in the azimuthal degree of freedom. They appear to bear the signature of parton-parton collisions. Thus, both string fragmentation and parton-parton hard scattering lead to clusters. One expects intuitively that the string fragmentation will likely lead to a chain of hadrons all along the rapidity axis as in source 1 in event 5 and source 2 in event 6 , whereas a parton-parton hard scattering will lead to two groups of clusters apart in rapidity, as in sources 2 in event 5 , and source 1 in event 6 .

\section{ALGORITHM FOR FINDING CLUSTERS}

As discussed in Sec. II, a minijet shows up as a cluster of hadrons with a cone radius of $R=0.6$ in the $(\eta, \phi)$ space. The sample events in PYTHIA6.4 in Sec. III indicate that there are clusters of produced particles in PYTHIA model calculations. Minijets are theoretically presumed to be produced in PYTHIA calculations. It is, therefore, useful to look for clusters as possible candidates for minijets.

Clusters, of the type shown in the last sections, can be searched for by the k-means clustering method [85-91], in conjunction with an additional k-number (cluster-number) specification principle. In such a search, we ascribe the characteristic cluster radius $R=0.6$ to a cluster. If the minijet producing hard-scattering process is dominant in the low- $p_{T}$ region, as suggested in earlier studies [26,29,55-58], then two clusters that are azimuthally correlated in a back-to-back manner have a high probability to be a physical mini-dijet of two correlated minijets at high collision energies.

For a given set of $M$ produced particles specified by their angular positions $\left\{\boldsymbol{x}_{i}=\left(\eta_{i}, \phi_{i}\right), i=1,2,3, \ldots M\right\}$ and a given $K$ number of clusters, the k-means clustering method consists of (i) partitioning the set of $M$ particles into $K$ cluster subsets $S_{k}=\left\{\boldsymbol{x}_{i}^{k}\right\}, k=1,2, \ldots, K$ and (ii) finding for each cluster subset the corresponding cluster center $\left\{\boldsymbol{C}_{k}, k=1,2, \ldots K\right\}$ so as to minimize the potential function

$$
\Phi(K)=\sum_{k=1}^{K}\left\{\sum_{\boldsymbol{x}_{i}^{k} \in S_{k}}\left(\boldsymbol{x}_{i}^{k}-\boldsymbol{C}_{k}\right)^{2}\right\},
$$

which is defined as the total subset sum of the squares of the distances between the cluster subset points and their corresponding cluster center $\boldsymbol{C}_{\boldsymbol{k}}$.

For a fixed value of $K$, the variation of the above potential function $\Phi(K)$ with respect to the cluster center $\boldsymbol{C}_{k}$ is given by

$$
\delta \Phi(K)=-\sum_{k=1}^{K}\left\{\sum_{\boldsymbol{x}_{i}^{k} \in S_{k}} 2\left(\boldsymbol{x}_{i}^{k}-\boldsymbol{C}_{k}\right) \cdot \delta \boldsymbol{C}_{k}\right\} .
$$

Because all $\delta C_{k}$ are independent, the minimization of $\Phi(K)$ with respect to the variation of the positions of the cluster centers $\boldsymbol{C}_{k}$ leads to $\delta \Phi(K) / \delta \boldsymbol{C}_{k}=0$ and

$$
\sum_{\boldsymbol{x}_{i}^{k} \in S_{k}} 2\left(\boldsymbol{x}_{i}^{k}-\boldsymbol{C}_{k}\right)=0 .
$$

This yields $\boldsymbol{C}_{k}$ as the centers of gravity of the subset of points of $S_{k}=\left\{\boldsymbol{x}_{i}^{k}\right\}, k=1,2, \ldots, K$,

$$
\boldsymbol{C}_{k}=\frac{1}{M_{k}} \sum_{\boldsymbol{x}_{i}^{k} \in S_{k}} \boldsymbol{x}_{i}^{k},
$$

where $M_{k}=\left(\sum_{x_{i}^{k} \in S_{k}} 1\right)$ is the number (multiplicity) of particles in the subset $S_{k}$.

In numerical implementation of the k-means clustering method for a given value of the cluster number $K$, one chooses randomly the first cluster center as one of the data points and chooses randomly the other $K-1$ cluster centers in the other data points with probability proportional to the square of the distance from the first cluster center [89]. For each data point, the knowledge of the positions of the initial cluster centers then allows one to calculate the squares of the distance between the data point and all $K$ cluster centers. One then assigns each data point to the subset $S_{k}$ with the smallest square of distance to its cluster center $\boldsymbol{C}_{k}$. After all subset assignments to $S_{k}$ have been completed for all data points, the center of gravity of the data points in each new subset $S_{k}$ is then recalculated to give the new cluster centers $\boldsymbol{C}_{k}$, with which the iterative procedure will proceed until it converges. One then calculates the potential function $\Phi(K)$ of Eq. (3) as the sum of squared distances.

The above standard procedure is then repeated with other random initialization of the initial cluster centers. After many cluster center random initializations, corresponding convergent solutions, and the potential functions $\Phi(K)$ have been obtained, the proper solution for the case of a given value of $K$ can be found and selected as the solution with the minimum value of the potential function $\Phi(K)$. For a given value of $K$, the k-means clustering method then yields uniquely the cluster subsets of particles $S_{k}=$ $\left\{\boldsymbol{x}_{i}^{k}\right\}, k=1,2, \ldots, K$ associated with each cluster and the corresponding cluster center location $\boldsymbol{C}_{k}$.

The k-means clustering method needs an amendment to make it applicable for cluster searches because the method will lead to poorly displaced and inaccurate cluster centers, if isolated particle points that are obviously not part of a cluster and quite far away from a cluster have been included into the particle data set in the clustering algorithm. The presence of these isolated particles is possible because the cluster partners of these isolated particles may not be detected within the narrow window of acceptance, and there may further be other sources of particle production in 
addition to those from clusters. We need to use our knowledge on the structure of the minijet in Eq. (1) to sieve out these isolated data points in the set of $M$ particles. We calculate the distances between any data point and all other data points in the $(\eta, \phi)$ plane. The knowledge of these distances allows us to exclude any data point whose minimum separation to all other data points exceeds a distance $2 R$, presumably the maximum separation for two data points in a minijet. (If we allow a degree of fuzziness in excluding these isolated points, the minimum separation can be set to $2 R+2 a$, where $a \ll R$ is the diffuseness parameter.) After these points are excluded to yield a reduced set of particles belonging to clusters in this modification, the k-means clustering method becomes very efficient, fast converging, and capable of yielding accurate cluster centers. The method is stable against the variations of the positions of the cluster centers, which turn out to be the centers of gravity of the subset $S_{k}$ of the clustering points, as given by Eq. (6). In this procedure, because the azimuthal angle $\phi$ is equivalent to $\phi \pm 2 \pi$ with a modulo of $2 \pi$, it is important to wrap around the azimuthal angles when such a wrapping leads to an additional possibility of minijet clustering.

We presume on the outset that a cluster consists of at least two particles. The k-means clustering method requires a prior knowledge of the cluster number $K$. There may be different ways to partition a group of $M$ particles into different numbers of clusters, and the locations of the cluster centers may also vary. The selection of $K$ and the identification of particles as belonging to different $K$ clusters may, therefore, be ambiguous. Our algorithm to find clusters must contain an additional method to select the appropriate cluster number $K$ that is based on well-founded physical principles.

For a given set of $M$ produced particles on the $(\eta, \phi)$ plane, one considers a possible range of cluster $K$ numbers, $K=K_{\min }, \ldots, K_{\max }$. The maximum limit $K_{\max }$ occurs when the cluster number $K_{\max }+1$ leads to the forbidden case of having a cluster with only a single particle. For each cluster number $K$ in the range under consideration, the k-means clustering method leads to a unique partition into $K$ clusters with their corresponding cluster centers $\boldsymbol{C}_{k}$. To select the appropriate $K$, we use the minijet physical properties discussed in the last section that a cluster circle with a radius $R=0.6$ of a physical minijet contains almost all of the particles of the physical minijet. In order for the cluster number $K$ to lead to the appropriate partition of the set of $M$ particles into $K$ physical minijets or clusters, the corresponding $K$ cluster circles with a radius $R=0.6$ should contain all, or almost all, $M$ data points of the set. There should be very few data points outside the cluster circles. The k-number (cluster number) selection principle is, therefore, that $K$ should be the cluster number that leads to the fewest number of data points $\Omega$ outside the cluster circles with an assumed radius.
In the process of determining quantitatively the number of outside data points, one finds that there are often some data points close to the circular boundary, which can be considered as part of the cluster. To account for such a possibility of inclusion of these hadrons into the clusters, we generalize the number of outside points from a discrete number $\Omega$ to a continuous quantity by

$$
\Omega=\sum_{k=1}^{K}\left\{\sum_{\boldsymbol{x}_{i}^{k} \in S_{k}}\left[1-\frac{1}{1+\exp \left\{\frac{\left|\mathbf{x}_{i}^{k}-\mathbf{C}_{k}\right|-R}{a}\right\}}\right]\right\},
$$

where, for our case, we have taken the value $R=0.6$. In the case with a sharp boundary $a \rightarrow 0$, we just have the case of a discrete number of outside points. We shall use $a=0.1$ for numerical purposes. In applying the principle of the least number of outside points, we calculate the generalized $\Omega$ only for points close to the cluster's boundary with the region between $R \sim R+a$. We directly reject points beyond $R+a$ as they are too far away from the clusters, and the possibility to involve these points inside the clusters is also very low. What is more, we also directly involve the points within $R$ inside the clusters. By these ways, we can make the sharp circle clusters to be flexible, and we still ensure the algorithm to be stable and fast.

For each iteration in each event, there may be particles farther away from all cluster centers beyond the separations of $R+a$ after particles are partitioned into sets of clusters. These data points will not be included in the determination of the new cluster centers for the next iteration.

By generalizing the number of outside points $\Omega$, from a discrete number to a continuous quantity, the principle of smallest outside points choice of $K$ is such that $K$ is that the quantity $\Omega$ is smallest for different $K$. If there are two $K$ values having the same fewest outside points within a range, we should select the smaller $K$ value because the set of the smaller number of minijets can radiate a parton and become the parent of the set with a greater number of minijets.

In summary, our cluster finding algorithm, therefore, consists of the k-means cluster method, supplemented by the k-number selection principle of the fewest number of data points outside of the cluster circles.

\section{ILLUSTRATION OF THE ALGORITHM FOR FINDING CLUSTERS}

We shall apply the above algorithm for finding clusters from charged hadrons generated by the PYTHIA8.1 for highenergy $p p$ collisions at $\sqrt{s}=200 \mathrm{GeV}$. The event generators PYTHIA8.1 [9] and PYTHIA6.4 [8] include the multiple parton interaction processes as described in Ref. [7] with additional considerations on color correlations, flavor correlations, junction topology, beam remnant configurations [11], and interleaving initial state radiations [12]. The 
fully interleaving evolution [13] and rescattering [14] are further included in PYTHIA8.2 [10].

In the series of PYTHIA programs, the basic picture of the multiple collision process arises from the composite nature of the proton, which possesses a parton spatial distribution in addition to the standard parton momentum distribution (parton distribution function). The parton-parton collisions between the constituents of the projectile proton and the target proton are assumed to be independent of each other, and the number of collisions in an event is, therefore, given by a Poisson distribution. The probability of parton-parton collisions is then a function of the parton-parton cross section and the impact parameter. To extend the partonparton scattering cross section to the low- $p_{T}$ region for minimum-bias studies, the divergent parton-parton scattering cross section at low transverse momenta has to be regularized with a cut-off parameter that can be chosen to yield the appropriate charged-hadron multiplicity distribution. We expect finite multiple parton-parton multiple collision probabilities for the independent collisions of projectile partons with target partons as depicted in the diagrams in Fig. 1. They lead to the production of multiple minijets and mini-dijets in the angular scatter plots of produced charged particles.

The probability for the occurrence of minijets and minidijets depends on the charge multiplicity of the event, which is part of the total hadron multiplicity. For brevity of notation and its frequent usage, we shall abbreviate "charge multiplicity" or "charged-particle multiplicity" simply by "multiplicity" when ambiguities do not arise or are not pertinent. We can restore back the term "charge multiplicity" when it is properly needed.

In order to predict what may be expected experimentally for multiple minijet and mini-dijet productions, we generate minimum-bias events using the PYTHIA8.1, and we accept primary charged particles with $|\eta| \leq 1$. For each event multiplicity, we select five random events for illustration. We shall label each event by the index $\mathrm{p} M \mathrm{e} I$, where $\mathrm{p} M$ stands for PYTHIA minimum-biased events with charge multiplicity $M$, and e $I$ denotes event number $I$ with the charge multiplicity $M$. We would like to search for the presence of the expected and mini-dijetlike clusters from the angular scatter plots of charged particles in these events.

The detected and identified charged particles include not only charged hadrons but also a small percentage (of about $12 \%)$ of $e^{+}$or $e^{-}$. By convention, we include these leptons in our charged multiplicity counts. However, because the $e^{+}$and $e^{-}$particles arise from many different hadronic and nonhadronic sources and the relations between these particles and their hadron parents if they arise from hadronic decays are nontrivial, we shall exclude them in our minijet finding algorithm. Their presence in the scattered $(\eta, \phi)$ plot provides a sense of possible hadronic activities in the vicinity of their angular locations.
In Figs. 4, 6, 7, and 8, we shall show sample scatter plots of charged particles in the $(\eta, \phi)$ plane from minimum-bias events simulated by the PYTHIA8.1 event generator. We display the particle labels of kaons, protons, electrons, and muons while the other particles are all charged pions. The solid and open points denote positive and negative particles, respectively, and circular and square points denote $p_{T} \leq 0.5 \mathrm{GeV} / \mathrm{c}$ and $p_{T}>0.5 \mathrm{GeV} / \mathrm{c}$, respectively.

We shall illustrate the algorithm for finding clusters with concrete examples. We consider three randomly selected minimum-bias PYTHIA8.1 events with $M=20$ in Fig. 4. For each of these events, we assume different cluster numbers $K$ and obtain $K$ clusters and their corresponding cluster centers $\boldsymbol{C}_{k}$ using the k-means clustering method. We then construct cluster circles with a radius $R=0.6$ circumscribing the cluster centers.

In Fig. 4, for event p20e 2 with $K=4,5,6$, and 7 on the top panel, the number of points $\Omega$ outside of the cluster circles are 10, 6, 4, and 2, respectively. For the case of $K=8$, there is no k-means clustering solution without one of the clusters possessing only a single particle. Because we do not consider a single particle to be a cluster, $K=8$ is excluded from our consideration for event $\mathrm{p} 20 \mathrm{e} 2$. If the clusters are minijet clusters, then almost all particle points should be inside the cluster circles. The case of $K=7$ leads to the fewest number of particles $\Omega$ outside of the cluster circles. According to the principle of fewest outside points, $K=7$ is the proper number of clusters for event $\mathrm{p} 20 \mathrm{e} 2$ on the top panel. Similarly, for event p20e4 in Fig. 4 with $K=4,5,6$, and 7 in the middle panel, the number of points outside of the cluster circles are 8, 5, 3, and 0 , respectively. We infer that $K=7$ leads to clusters for event p20e4. For event p20e5 in Fig. 4 with $K=3,4,5$, and 6 in the lower panel, the number of outside points are $11,6,1$, and 0 . We infer that $K=6$ is the proper cluster number with zero points outside of the cluster circles.

It should be mentioned that there is another method, the "elbow method", to select the cluster number $K$ by studying the $K$-dependence of the potential function $\Phi(K)[85,90]$. The method consists of determining the cluster number by the location of the "kink" where there is a sudden change of the slope of the potential function. The method suffers from the ambiguities in finding where the kink lies and will not be used in the present context. We shall discuss the ambiguities in such a method in Appendix A.

\section{SCATTER PLOTS OF PRODUCED CHARGED PARTICLES FROM PYTHIA8.1}

We study the clustering properties of charged particles produced in $p p$ collisions in events with $-1 \leq \eta \leq 1,-\pi \leq$ $\phi \leq \pi$ and generated by PYTHIA8.1 at $\sqrt{s}=200 \mathrm{GeV}$ without a $p_{T}$ selection. In reviewing the scatter plots in the $(\eta, \phi)$ space as a function of the charged particle multiplicity, it should be kept in mind that those events with larger charge multiplicity numbers $M$ are events with 

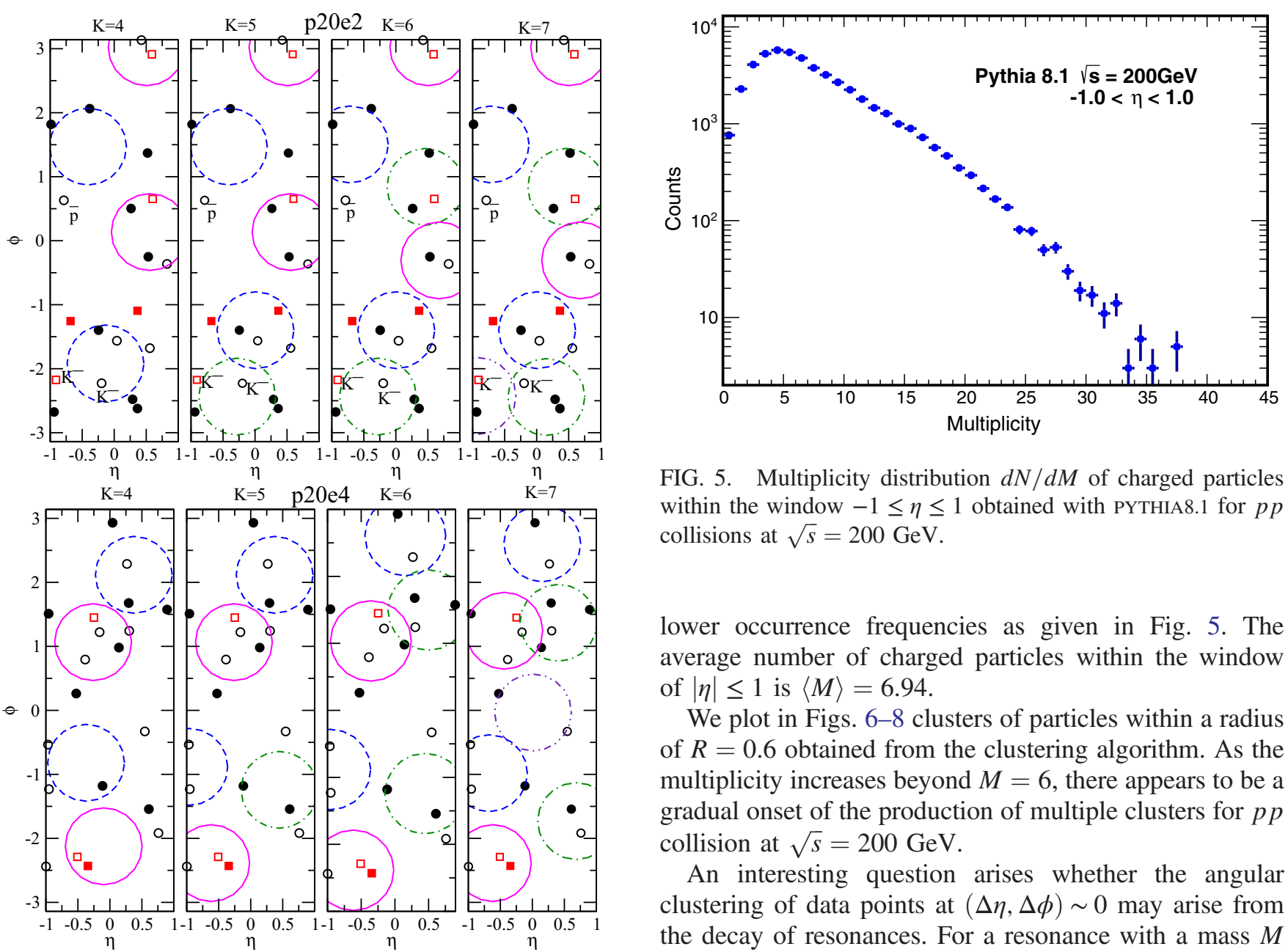

FIG. 5. Multiplicity distribution $d N / d M$ of charged particles within the window $-1 \leq \eta \leq 1$ obtained with PYTHIA8.1 for $p p$ collisions at $\sqrt{s}=200 \mathrm{GeV}$.

lower occurrence frequencies as given in Fig. 5. The average number of charged particles within the window of $|\eta| \leq 1$ is $\langle M\rangle=6.94$.

We plot in Figs. 6-8 clusters of particles within a radius of $R=0.6$ obtained from the clustering algorithm. As the multiplicity increases beyond $M=6$, there appears to be a gradual onset of the production of multiple clusters for $p p$ collision at $\sqrt{s}=200 \mathrm{GeV}$.

An interesting question arises whether the angular clustering of data points at $(\Delta \eta, \Delta \phi) \sim 0$ may arise from the decay of resonances. For a resonance with a mass $M$ decaying into two particles with momenta $p_{i}=\left(y, p_{T i}, \phi_{i}\right)$ with $i=1,2$ and transverse masses $m_{T i}=\sqrt{p_{T i}^{2}+m_{i}^{2}}$, the angular correlation of the two particles for $\Delta y=\left(y_{1}-y_{2}\right)$ and $\Delta \phi=\phi_{1}-\phi_{2}$ satisfies

$$
\begin{aligned}
& \frac{M^{2}-m_{1}^{2}-m_{2}^{2}}{2} \\
& \quad=m_{T 1} m_{T 2} \cosh (\Delta y)-p_{T 1} p_{T 2} \cos (\Delta \phi) .
\end{aligned}
$$

For small $|\Delta y|$ and $|\Delta \phi|$, we can expand the cosh and cos functions and get

$$
\begin{aligned}
& m_{T 1} m_{T 2}(\Delta y)^{2}+p_{T 1} p_{T 2}(\Delta \phi)^{2} \\
& \quad=M^{2}-m_{1}^{2}-m_{2}^{2}-2 m_{T 1} m_{T 2}+2 p_{T 1} p_{T 2} .
\end{aligned}
$$

particles in minimum-bias events with multiplicities $M=20$ for $-1 \leq \eta \leq 1$, in sample events p20e2, p20e4, and p20e5 generated by PYTHIA8.1 for $p p$ collisions at $\sqrt{s}=200 \mathrm{GeV}$. Cluster circles with a radius $R=0.6$ circumscribe cluster centers obtained with the k-means clustering method assuming different cluster numbers $K$.

In the decay into two masses, the scatter plot of the two final particles for small values of $\Delta y$ and $\Delta \phi$ fall within an ellipse with ellipsoidal radii given by 

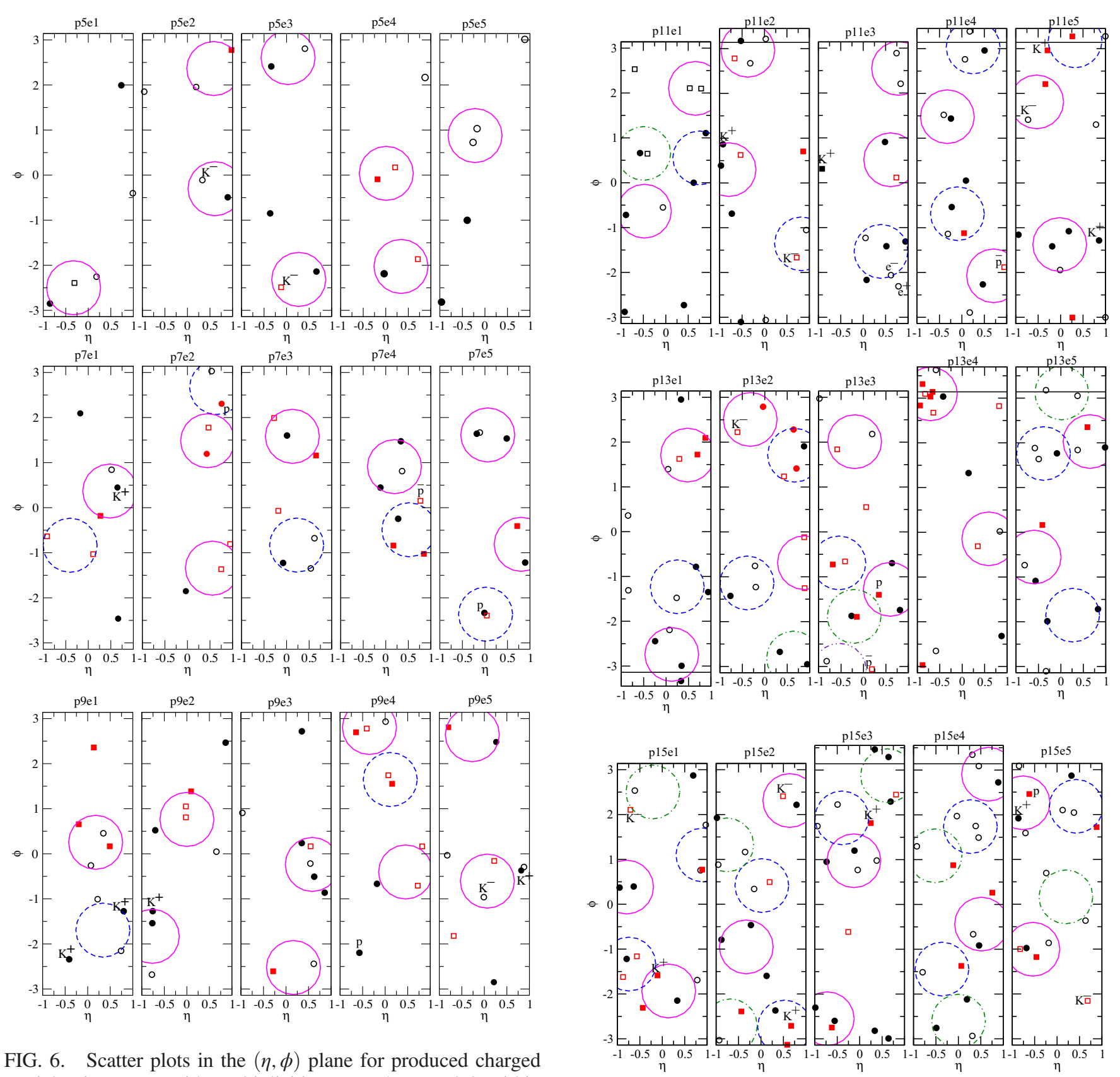

FIG. 6. Scatter plots in the $(\eta, \phi)$ plane for produced charged particles in events with multiplicities $M=5,7$, and 9 within $-1 \leq \eta \leq 1$ generated by the PYTHIA8.1 for $p p$ collisions at $\sqrt{s}=200 \mathrm{GeV}$. Circular curves indicate the locations of the clusters.

$$
\begin{aligned}
& a_{\Delta y}=\sqrt{\frac{M^{2}-m_{1}^{2}-m_{2}^{2}-2\left(m_{T 1} m_{T 2}-p_{T 1} p_{T 2}\right)}{m_{T 1} m_{T 2}}}, \\
& a_{\Delta \phi}=\sqrt{\frac{M^{2}-m_{1}^{2}-m_{2}^{2}-2\left(m_{T 1} m_{T 2}-p_{T 1} p_{T 2}\right)}{p_{T 1} p_{T 2}}} .
\end{aligned}
$$

Thus, the decay of a resonance may appear as a cluster within a radius $a_{\Delta y}$ and $a_{\Delta \phi}$ and not necessarily and directly from a minijet, depending on the quantities as given on the

FIG. 7. Scatter plots in the $(\eta, \phi)$ plane for $M=11,13$, and 15 , within the window of $-1 \leq \eta \leq 1$ generated by the PYTHIA8.1 for $p p$ collisions at $\sqrt{s}=200 \mathrm{GeV}$. Circular curves indicate the locations of the clusters.

right-hand side of the above equations. Upon approximating the rapidity $y$ as the pseudorapidity $\eta$, the above results show that the decay of a resonance may appear as a cluster with the radii of Eq. (10).

The partitioning of the set of charged particles into clusters can be carried out on an event-by-event basis in Figs. 6-8 by identifying a cluster as an assemble of particles represented by a circle in the $(\eta, \phi)$ plane with a radius of $R=0.6$. We can furthermore identify a 


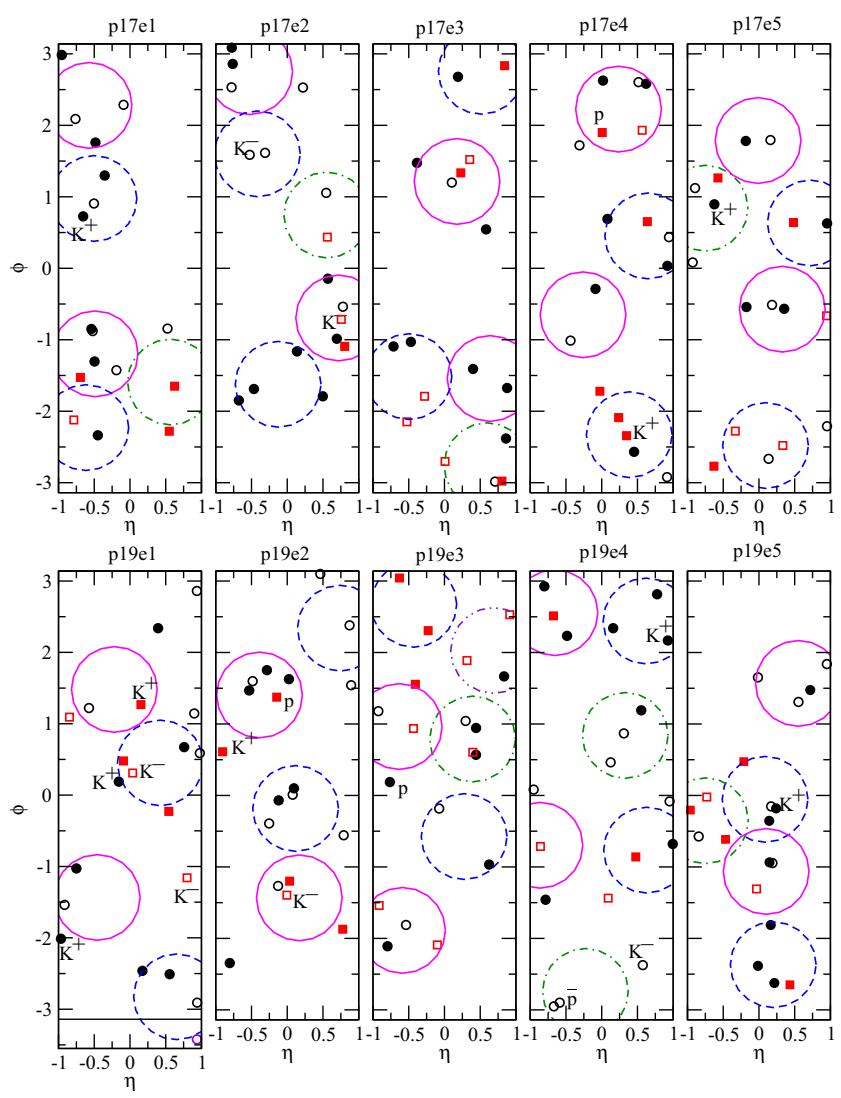

radius of $R=0.6$ occur with a greater probability. In most of the events with $M=7$ to 9 and higher multiplicities, a single cluster appears often to correlate roughly with an associated partner in azimuthally nearly back-to-back directions. There may be a fluctuation of the back-to-back correlation due to the intrinsic transverse momentum of the partons. We conclude from these figures that mini-dijetlike clusters commence at $M \sim 7$ with the probability increasing gradually as $M$ increases and appear nearly consistently for $M \gtrsim 11$, as indicated in Figs. 7 and 8.

We show in Fig. 7 the scatter plots of charged particles in events with high multiplicities $11 \leq M \leq 15$. As the multiplicity number $M$ increases beyond $M \gtrsim 13$, there is a transition from the production of one pair of minidijetlike clusters to the production of two pairs of minidijetlike clusters with each pair of mini-dijetlike clusters approximately azimuthally back-to-back with respect to each other. The transition region is not sharp as many events contain only a single pair of mini-dijetlike clusters while many other events in Fig. 7 contain double correlated mini-dijetlike clusters. We conclude from these figures that two mini-dijetlike cluster pairs begin to set in with $M \gtrsim 14$ with the probability increasing gradually as $M$ increases.

We show in Figs. 8 the scatter plots of charged particles in events with ultra-high multiplicities $17 \leq M \leq 21$. As the multiplicity number $M$ increases beyond $M \gtrsim 17$, the production of two sets of mini-dijetlike clusters appears nearly consistently with occasional production of five clusters. In Fig. 8, events with $M \gtrsim 20$ appear to contain events with three pairs of mini-dijetlike clusters.

The results from the present analysis indicates that multiple clusters and mini-dijetlike clusters are common occurrences for events with high multiplicities, and their numbers increase with the increasing multiplicity $M$.

Figure 9(a) shows that for events generated by PYTHIA8.1 within $|\eta| \leq 1$, the number of clusters $K$ appears to increase monotonically and approximately as a linear function of charge multiplicity $M$. The relationship between $(M / K)_{\text {PYTHIA }}$ and $M$ is shown in Fig. 9(b). The ratio $(M / K)_{\text {PYTHIA }}$ is 2.355 for $M=5$ and is 2.372 for $M=8$.

\section{CLUSTERING OF PARTICLES IN A RANDOM DISTRIBUTION}

within the window of $-1<\eta<1$ generated by the PYTHIA8.1 for $p p$ collisions at $\sqrt{s}=200 \mathrm{GeV}$. Circular curves indicate the locations of the clusters.

mini-dijetlike pair of clusters as two correlated clusters whose centers are separated azimuthally within the range from $\pi-R$ to $\pi+R$. In Figs. 6-8, we indicate a cluster and its corresponding associated partner by circles of the same line type and color. At the end edges of $\phi= \pm \pi$, the scatter plots are sometimes wrapped around so as to facilitate the partitioning particles into clusters, as in events p11e2, p11e4, p11e5,...

The data in Figs. 6-8 reveal that as the multiplicity increases, clusters of more than two particles within a

The results in the last section indicate the copious production of clusters in the theoretical model of PYTHIA8.1. Many of these clusters also exhibit back-toback azimuthal correlations to make them good candidates for physical mini-dijets. These theoretical clusters as well as their corresponding experimental counterparts will likely represent physical minijets and mini-dijets, if the dominance of the parton-parton hard-scattering process for minijet production is extended to the low- $p_{T}$ region as suggested in $[26,29,55-58]$.

It is worth noting that the clustering property by itself is not sufficient to definitively identify a cluster as a minijet 

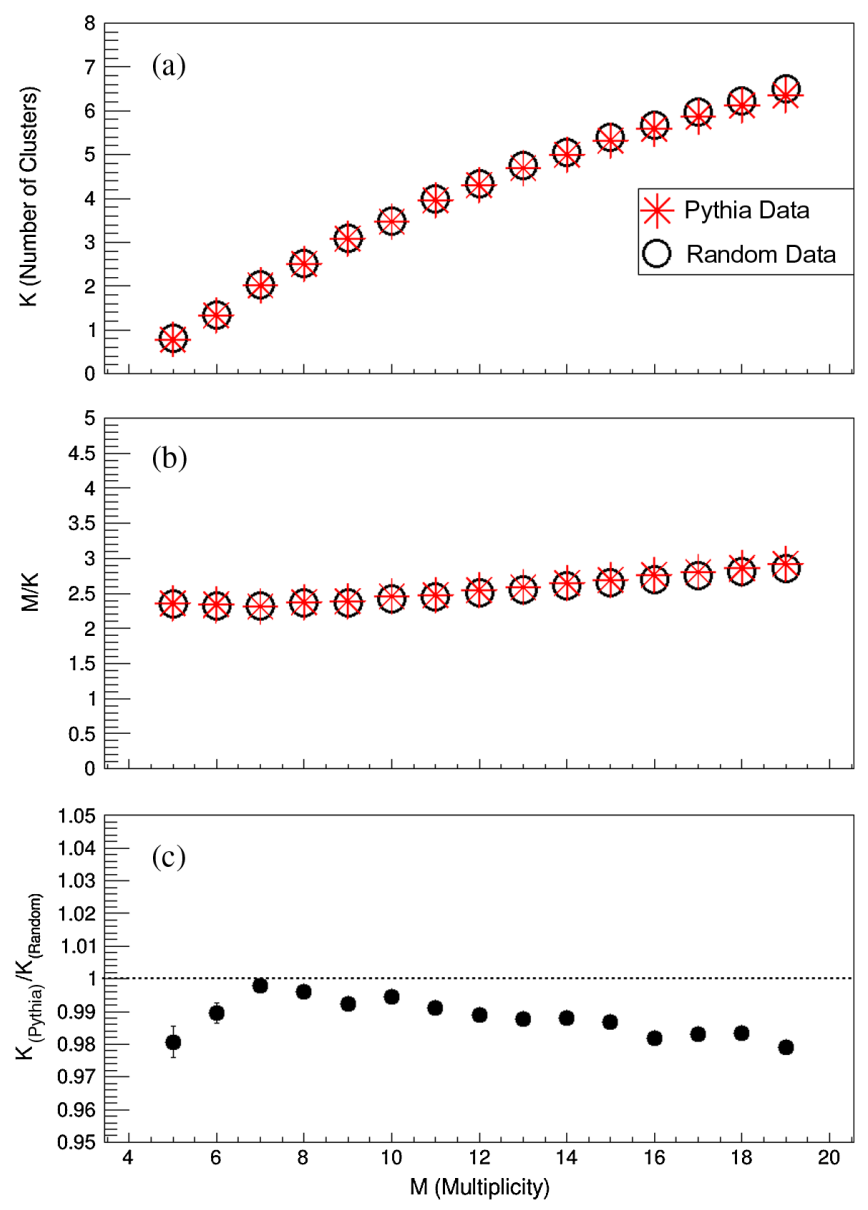

FIG. 9. Relations between charge multiplicity $M$ and the number of clusters $K$ : for $p p$ collisions at $\sqrt{s}=200 \mathrm{GeV}$ as extracted from events generated by PYTHIA8.1 within $|\eta| \leq 1$ and $|\phi| \leq \pi$. (a) The relation of $K$ as a function of $M$ obtained from PYTHIA (star symbol) and the random distribution (open circle). (b) The ratio of $K / M$ as a function of $M$ for particles. (c) The ratio of $K_{\text {PYTHIA }} / K_{\text {random }}$ as a function of $M$.

cluster because similar clustering properties may also be present in other particle production models. It is necessary to have other independent collaborative supports for the minijet occurrence in order to identify the observed clusters as likely physical minijet clusters.

In order to bring the need for independent collaborative supports into sharp focus, it is illustrative to examine the clustering properties of particles produced in a simple schematic model in which a total of $M_{\text {random }}$ number of particles are randomly and independently produced with a uniform probability in the $(\eta, \phi)$ phase space within the window of $|\eta| \leq \Delta \eta_{\text {window }} / 2$ and $|\phi| \leq \pi$,

$$
\frac{d P_{\text {random }}}{d \eta d \phi}=\frac{\Theta\left(\Delta \eta_{\text {window }} / 2-|\eta|\right) \Theta(\pi-|\phi|)}{2 \pi \Delta \eta_{\text {window }}} .
$$

This can be the approximate mode of production when particles are produced independently with a uniform probability in rapidity, as from the fragmentation of a flux tube at very high energies $[6,10,25,30-46]$. It can also be the probability distribution used to describe noise particles randomly produced within the experimental $(\eta, \phi)$ phase space. We use the symbols $\left\{M_{\text {random }}, K_{\text {random }}\right\}$ to denote the multiplicity number and cluster numbers, respectively, using a random generation of particles.

We find, in this case of random distribution, that particle clustering also occurs when a large $M_{\text {random }}$ number of particles are produced randomly over a small phase space. To understand such a clustering, we can pick any two produced particles. The probability that a pair of particles falling randomly within the circle of radius $R$ with respect to each other is

$$
P_{\text {random }}=\left(\frac{\pi R^{2}}{\Delta \phi_{\text {window }} \Delta \eta_{\text {window }}}\right) \text {, }
$$

where $\Delta \phi_{\text {window }}=2 \pi$ and $\Delta \eta_{\text {window }}=2$ for the present window. In an event with multiplicity $M_{\text {random }}$, the number of distinct pairs is

(number of distinct pairs $)=\frac{M_{\text {random }}\left(M_{\text {random }}-1\right)}{2}$.

Therefore, with multiplicity $M_{\text {random }}$ the (average) number of clusters $K_{\text {random }}\left(2, M_{\text {random }}\right)$ is the product of Eqs. (12) and (13),

$K_{\text {random }}\left(2, M_{\text {random }}\right)=\frac{M_{\text {random }}\left(M_{\text {random }}-1\right)}{2\left(2 \pi \Delta \eta_{\text {window }}\right)} \pi R^{2}$,

upon identifying a cluster as two particles falling within a radius of $R=0.6$. However, because clusters can be formed with more than two particles, the above quantity $K_{\text {random }}\left(2, M_{\text {random }}\right)$ represents only the upper limit of the number of clusters when particles fall into and join other clusters.

More generally, the number $K_{\text {random }}\left(n, M_{\text {random }}\right)$ of clusters of random coincidence for a cluster of $n$ particles within a radius of $R$ in an event with multiplicity $M$ is

$$
K_{\text {random }}\left(n, M_{\text {random }}\right)=C_{n}^{M_{\text {random }}}\left(\frac{\pi R^{2}}{2 \pi \Delta \eta_{\text {window }}}\right)^{n-1} .
$$

For a detector such as the STAR detector with a pseudorapidity window $\Delta \eta_{\text {window }}=2$, we have

$K_{\text {random }}\left(2, M_{\text {random }}\right)=\frac{M_{\text {random }}\left(M_{\text {random }}-1\right)}{2} \times 0.09$.

Thus, the upper limit of the number of clusters from the random distribution Eq. (11) increases quadratically as a function of the multiplicity $M_{\text {random}}$. This upper limit can be quite large for large $M_{\text {random}}$. For example, from Eq. (16) one expects the upper limit of $K_{\text {random }}\left(2, M_{\text {random }}\right)=0.9$ 
and 4.95 clusters for $M_{\text {random }}=5$ and $M_{\text {random }}=11$, respectively. Thus, we would not be surprised to find clusters even for randomly and independently distributed particles as the multiplicity $M_{\text {random }}$ increases from 4 to 11 .

In our numerical example, we generate particles randomly with the uniform probability distribution of Eq. (11) within $-\pi \leq \phi \leq \pi$ and $-1 \leq \eta \leq 1$. We label the events as $x M_{\text {random }} e I$ and show sample events with multiplicity from $M_{\text {random }}=5$ to $M_{\text {random }}=21$ in Figs. 10-12, where we shall not distinguish the charges and the types of particles. We then use the minijet finding algorithm of Secs. III and IV to locate cluster centers and circumscribe the cluster in circles.

Figures 10-12 show that as the multiplicity increases, the number of clusters $K_{\text {random }}$ also increases. In Fig. 9(a), we show that the number of clusters $K_{\text {random }}$ appears to be nearly a linear function of the multiplicity, similar to the relationship for events generated by PYTHIA8.1. The number of clusters $K_{\text {random }}$ estimated by Eq. (16) represents only an upper limit because a cluster with more than two particles can be formed in high multiplicity events. The number of clusters increases only approximately linearly with multiplicity $M_{\text {random }}$ instead of the quadratic dependence of Eq. (13), as shown in Fig. 9(a).

Figure 9(a) shows the cluster numbers $K$ for events generated by the random distribution along with those generated by PYTHIA8.1 within $|\eta| \leq 1$. The number of clusters $K_{\text {random }}$ for the random distribution appears to increase, likewise, monotonically and approximately as a linear function of multiplicity $M=M_{\text {random. }}$ The relationship between $(M / K)_{\text {random }}$ and $M$ is shown in Fig 9(b). The ratio $(M / K)_{\text {random }}$ is 2.354 for $M=5$ and is 2.357 for $M=8$. The ratio $K_{\text {PYTHIA }} / K_{\text {random }}$ is close to unity. It is 0.981 for $M=5$, and it is 0.996 for $M=8$, as shown in Fig. 9(c).

One way to study the clusters that are formed is by way of the $\left(\Delta \eta=\eta_{1}-\eta_{2}, \Delta \phi=\phi_{1}-\phi_{2}\right)$ correlations between clusters located at $\left(\eta_{1}, \phi_{1}\right)$ and $\left(\eta_{2}, \phi_{2}\right)$. Figures $10-12$, for the random and uniformly distributed particles, also exhibit azimuthal correlations for some of the pairs, as cluster circles of similar types in these figures indicate. Thus, the clusters in the random distribution also exhibit approximate azimuthal back-to-back correlations, as can be observed in Figs. 10 and 11.

We can estimate the number of azimuthally back-to-back correlated clusters $D^{\prime}$ as a function of the number of clusters $K_{\text {random }}$. We consider a pair of clusters. The probability that the pair of clusters can be considered back-to-back correlated in azimuthal angles is

$$
P_{\text {random }}=\frac{2 R}{\Delta \phi_{\text {window }}}=\frac{2 R}{2 \pi} \text {. }
$$

In an event with $K_{\text {random }}$ number of clusters, the number of distinct pairs is
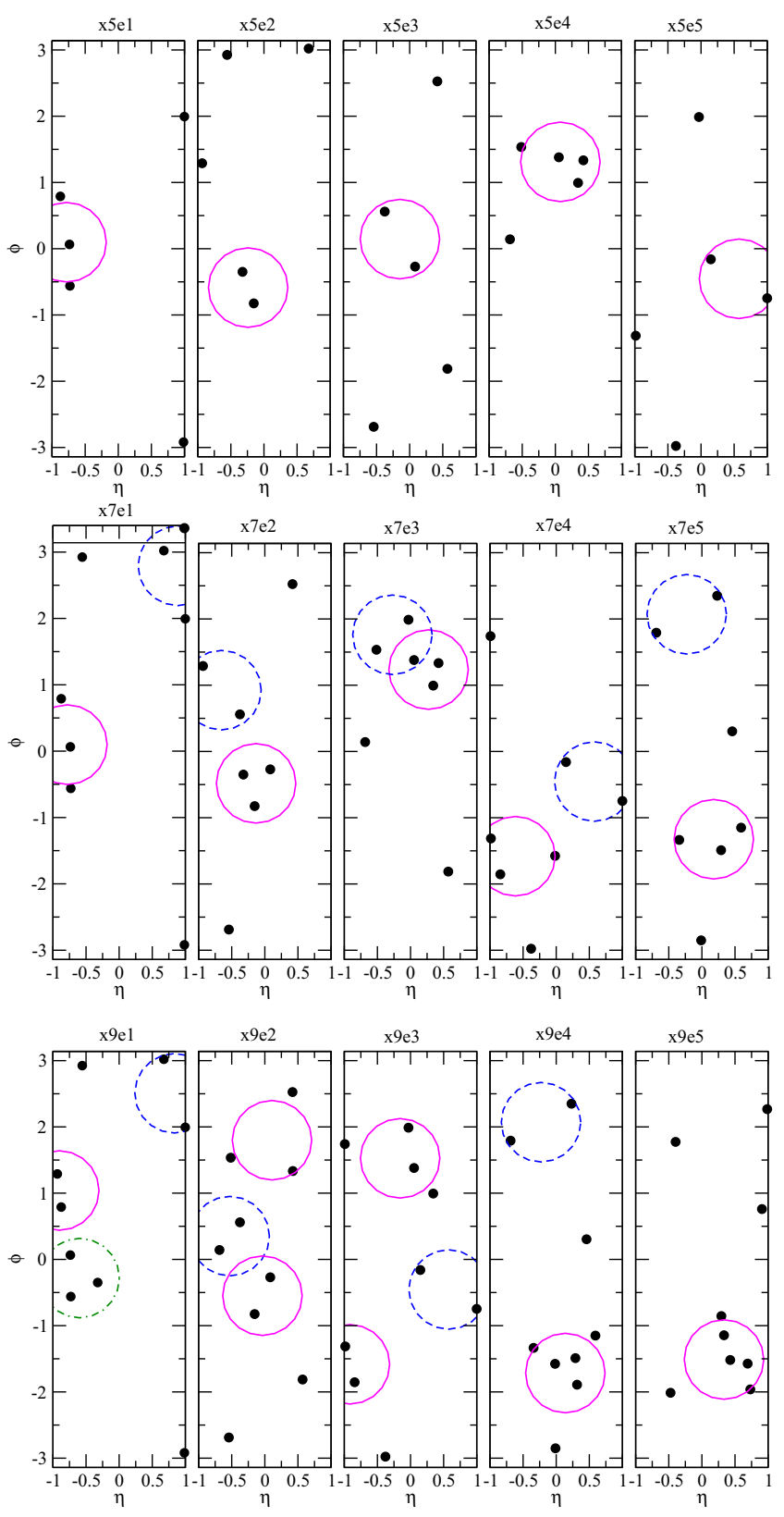

FIG. 10. Scatter plots in the $(\eta, \phi)$ plane for produced particles in events with multiplicities $M_{\text {random }}=5,7$, and 9, production within $|\eta| \leq 1$ and $|\phi| \leq \pi$. Circular curves indicate the locations of the cluster circles with $R=0.6$.

(number of distinct pairs $)=\frac{K_{\text {random }}\left(K_{\text {random }}-1\right)}{2}$.

Therefore, in such an event with $K_{\text {random }}$ number of clusters, the (average) number of mini-dijetlike pairs $D^{\prime}\left(K_{\text {random }}\right)$ for the random distribution is the product of Eqs. (14) and (18),

$$
D^{\prime}\left(K_{\text {random }}\right)=\frac{K_{\text {random }}\left(K_{\text {random }}-1\right)}{2}\left(\frac{R}{\pi}\right) .
$$



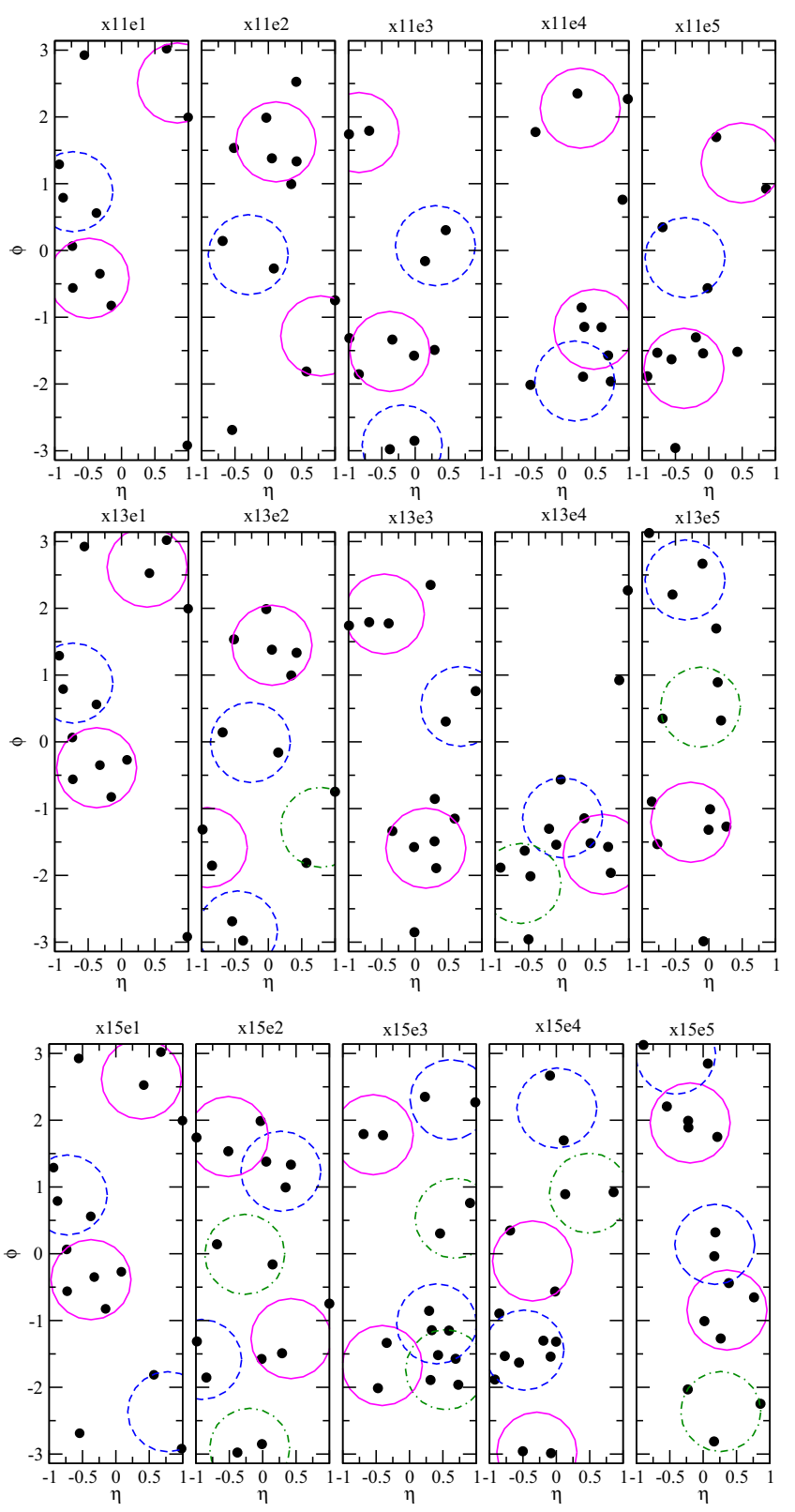

FIG. 11. Scatter plots in the $(\eta, \phi)$ plane for produced particles in events with multiplicities $M_{\text {random }}=11,13$, and 15 generated by an event generator with a uniform and independent production within $|\eta| \leq 1$ and $|\phi| \leq \pi$. Circular curves indicate the locations of the cluster circles with $R=0.6$.

Thus, for $K_{\text {random }}=4$, the number of mini-dijetlike pairs is $D^{\prime}\left(K_{\text {random }}\right)=1.15$. This means that when $K_{\text {random }}$ exceeds about four, the number of mini-dijetlike pair of clusters $D^{\prime} \sim 1$ and back-to-back correlated mini-dijetlike pairs will begin to set in, as one can observe from the number of minidijetlike clusters in events $\mathrm{x} 11 \mathrm{e} 3, \mathrm{x} 13 \mathrm{e} 2$, and $\mathrm{x} 15 \mathrm{e} 1$ with $K_{\text {random }} \gtrsim 4$ in Fig. 11.

Results in Figs. 10-12 indicate that by distributing particles densely within a small angular phase space,
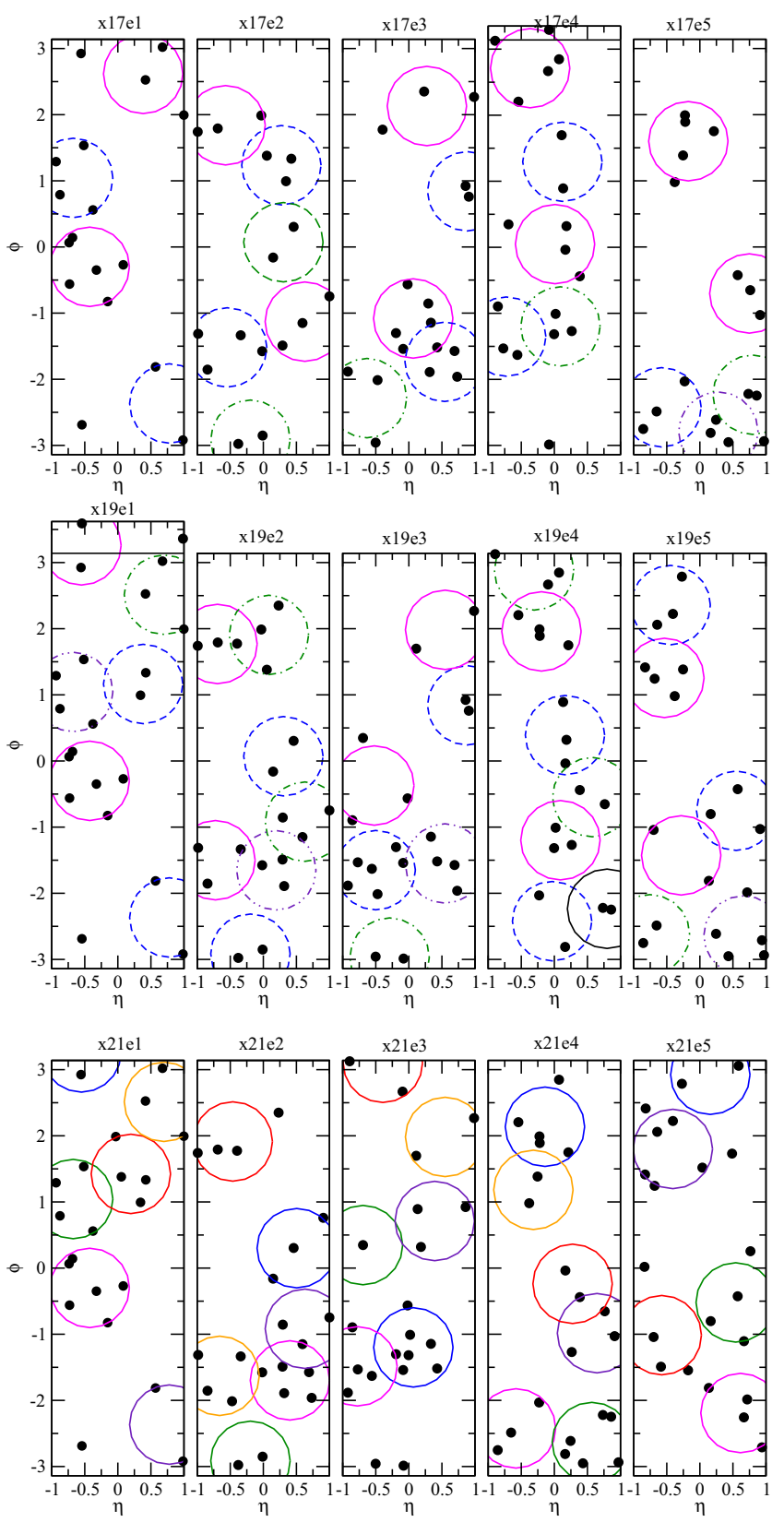

FIG. 12. Scatter plots in the $(\eta, \phi)$ plane for produced particles in events with multiplicities $M_{\text {random }}=17,19$, and 21 generated an event generator with a uniform and independent production within $|\eta| \leq 1$ and $|\phi| \leq \pi$. Circular curves indicate the locations of the cluster circles with $R=0.6$.

clustering and azimuthal correlations occur also for randomly distributed sources of the particle. Thus, clustering and azimuthal correlation by themselves cannot be the only means of identifying minijets and mini-dijets. The identification of these clusters, as such, arises from other independent supports for the dominance of the hard-scattering model for minijet production of low- $p_{T}$ particles. 


\section{CORRELATIONS BETWEEN PARTICLES AND BETWEEN CLUSTERS}

\section{A. Two-particle correlations}

The results in the previous sections indicate that while the PYTHIA event generator yields clusters in the $(\eta, \phi)$ plane, such a clustering is not uniquely a property of the dynamics of the particle production processes in QCD as implemented in PYTHIA. Clustering also occurs with randomly generated data arising from many sources. The angular dimension $\pi R^{2}$ relative to the window dimensions $\left.\Delta \eta\right|_{\text {window }}$ and $\left.\Delta \phi\right|_{\text {window }}$ gives rise to a finite clustering probability even for random distributions, as discussed in Eqs. (16), (17), and (19). The kinematic cuts and the shape of the kinematic window also plays a significant role. With a particular geometry in defining the acceptance window such that $\left\{\eta_{1}, \eta_{2}\right\} \in \Delta \eta_{\text {window }}$, the phase space of a correlated particle-particle pair will not always be distributed uniformly in the correlation coordinates $\{\Delta \eta, \Delta \phi\}$. As a consequence, the particle-particle correlations and the associated cluster-cluster correlations will be distorted. The assumed intrinsic property of the clusters play another important role. For example, if we set the $p_{T}$ acceptance threshold of the particles to be higher and higher, then the multiplicity number and the number of clusters will be lower, the greater the probability for a high multiplicity event to reveal itself much more readily as originating from a minijet as compared to a random cluster. Therefore, the meaning of a cluster is defined by a given set of the attributes of the delimiting constraints.

Given a set of these constraints, we would like to examine the particle-particle and cluster-cluster correlations for the purpose of extracting information on the dynamics that distinguishes the PYTHIA8.1 results from random results. The particle-particle and cluster-cluster correlations are also called two-particle and two-cluster correlations, respectively. After using PYTHIA8.1, or the random distribution to simulate $p p$ collisions, we collect the kinematic data of the particles in each event. We pick all combinations of particle pairs (or cluster pairs) that are in the same events to calculate the $\Delta \phi$ and $\Delta \eta$ between any two particles or clusters. Then we fill the 2D-histogram with $\Delta \phi$ and $\Delta \eta$ to get the particle-particle or cluster-cluster correlation function.

In Fig. 13, we show the un-normalized 2D two-particle correlation distribution $d N / d \Delta \eta d \Delta \phi$ as a function of the correlation separation $\Delta \eta$ and $\Delta \phi$ within the window of $-1 \leq \eta \leq 1$ and $0 \leq \phi \leq \pi$ for $M=5-7$. There is the symmetry of the distribution with respect to a change of the sign of $\Delta \phi$ or $\Delta \eta$. It suffices to display the distributions only in the region of positive $\Delta \phi$ and $\Delta \eta$. The color plot of the event number in each bin is red for large number counts and blue for fewer number counts.

The effect of the phase space limitations shows up clearly in the correlation function for the case of the
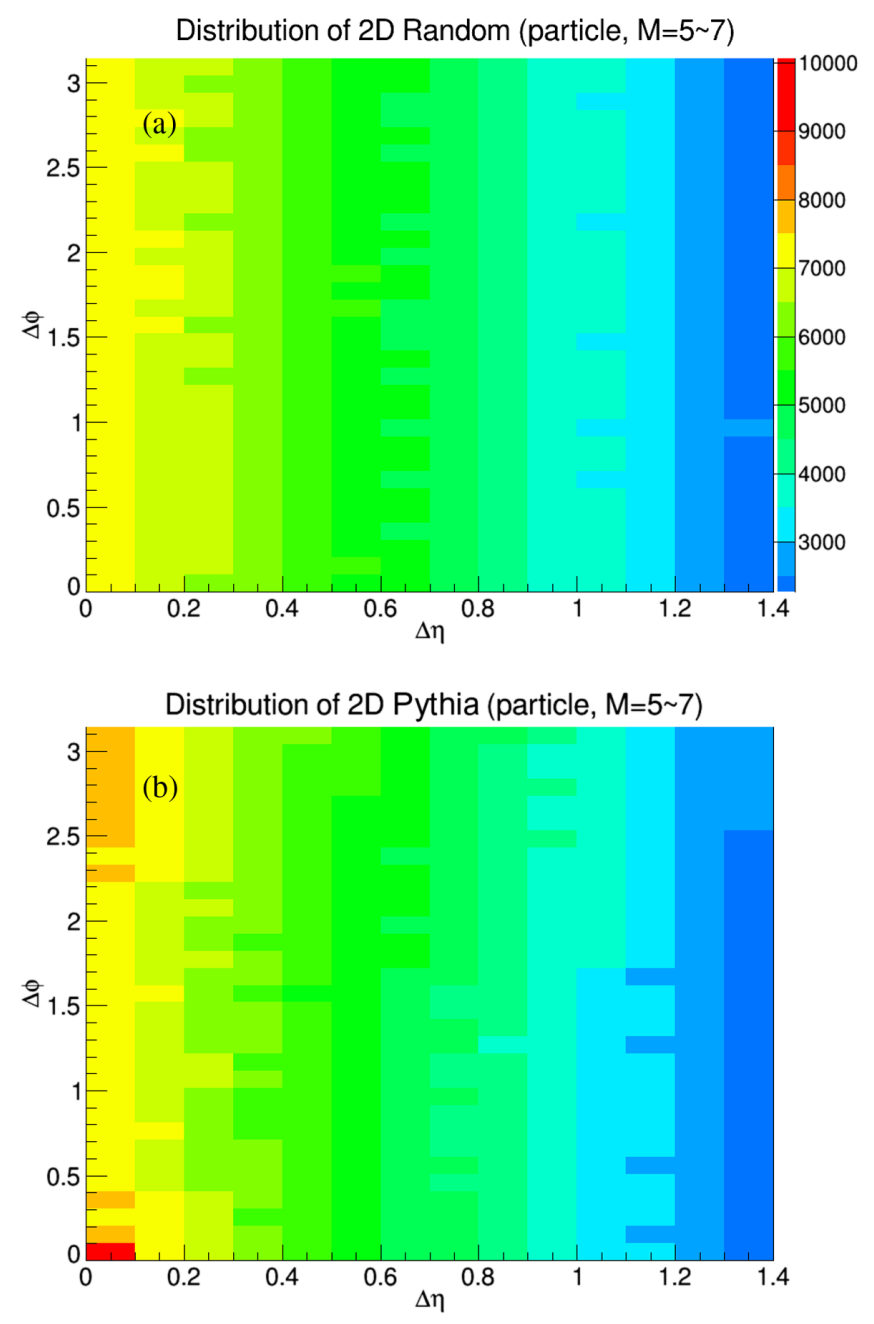

FIG. 13. Un-normalized 2D two-particle correlation distribution $d N /\left.(d \Delta \phi d \Delta \eta)\right|_{M}$ is produced by the events with multiplicities $M=5,6$, and 7. Figure (a) is the cluster-cluster correlation for the random events and Fig. (b) is the cluster-cluster correlation for PYTHIA events. The $\Delta \phi$ and $\Delta \eta$ are positive as they are only differences in the azimuthal and pseudorapidity coordinates.

random distribution in Fig. 13(a). Within the acceptance window, the generated correlation function $d N / d \Delta \phi d \Delta \eta$ along the $\eta$ axis is large at $\Delta \eta=0$, and it falls down linearly as $\Delta \eta$ increases in the well-known form of a triangular distribution. The generated correlated distribution is nearly uniform in the $\Delta \phi$ direction with minor fluctuations.

Figure 13(b) gives the two-particle correlation obtained with the event generator PYTHIA8.1 for $M=5-7$, which corresponds approximately to the average multiplicity $\langle M\rangle=6.94$. It represents essentially the theoretical particleparticle correlation function for the case of minimumbiased measurements. One notes that the limited phase space of the measurement window, likewise, distorts the distribution to follow roughly the triangular shapes as a function of $\Delta \eta$ with an approximately uniform distribution in $\Delta \phi$. However, upon careful examination, there are finer differences in the region of $(\Delta \phi, \Delta \eta) \sim 0$ and $\Delta \phi \sim \pi$. 
Along the $\Delta \phi$ axis, Fig. 13(b) shows two peaks at $\Delta \phi \sim 0$ and $\Delta \phi \sim \pi$.

The difference between the PYHTIA distribution and the random distribution shows up in sharper focus upon taking the ratio $d N /\left.d \Delta \eta d \Delta \phi\right|_{\text {PYTHIA }} / d N /\left.d \Delta \eta d \Delta \phi\right|_{\text {random }}$ at each $(\Delta \eta, \Delta \phi)$ point as shown in Fig. 14(a). We shall call such ratio, $d N /\left.d \Delta \eta d \Delta \phi\right|_{\text {PYTHIA }} / d N /\left.d \Delta \eta d \Delta \phi\right|_{\text {random }}$, the normalized correlation function. It is normalized with respect to the constraints of the measurement as represented by a random distribution within the measurement window. In the comparison with experimental data, such normalizations are often carried out by event-mixing data.

The normalized 2D particle-particle correlation obtained with the PYTHIA8.1 generator exhibits the gross feature of the well-known shape with a near-side peak at $(\Delta \eta, \Delta \phi) \sim 0$ arising from the near-side jet and an away-side ridge at $\Delta \phi \sim \pi$ along the direction of approximately constant $\Delta \eta$ by momentum conservation in a parton-parton collision $[55-63,80,92,93]$. Beyond these two regions, there is a region of low correlations with $\Delta \eta>0.6$ and $0<\Delta \phi<1.5$.

We can carry out similar analysis for other values of the multiplicity number $M$. We find out that particle-particle correlations change as the multiplicity $M$ increases. In Fig. 14(b), we show the particle-particle correlation for $M=11-13$, for which one finds that as the multiplicity increases the near-side jet gains in strength and angular size, and the away side correlation becomes weaker because momentum conservation is weakened by a larger multiplicity.

In Fig. 14(c), we show the particle-particle correlation for $M=17-18$, for which one finds that for such higher multiplicity events the near-side jet gains even greater in strength and angular size, and similar to the case of $M=11-13$ the away side ridge distribution cannot be distinguished.

\section{B. Two-cluster correlations}

While the particle-particle correlations comprise a part of the standard tools in the analysis of experimental data, it is of interest to develop cluster-cluster correlations as another useful tool in the study of the dynamics of the particle production process. Accordingly, for a set of delimiting cutoff attributes for accepting a particle and a cluster, we apply the cluster-searching algorithm to locate the clusters and their centers. Because each cluster has at least two particles, we start to study events with at least five particles so that there are sufficient number of particles and clusters to examine cluster-cluster correlations. With the knowledge of the cluster centers, we pick all combinations of particle pairs that are in the same event to calculate the $\Delta \phi$ and $\Delta \eta$ between the centers of any two clusters and obtain the cluster-cluster correlation.

Figure 15(a) is the 2D two-clusters correlation distribution of the $\Delta \phi$ and $\Delta \eta$ with $K=2$ for the random distribution. This case of $K=2$ corresponds approximately
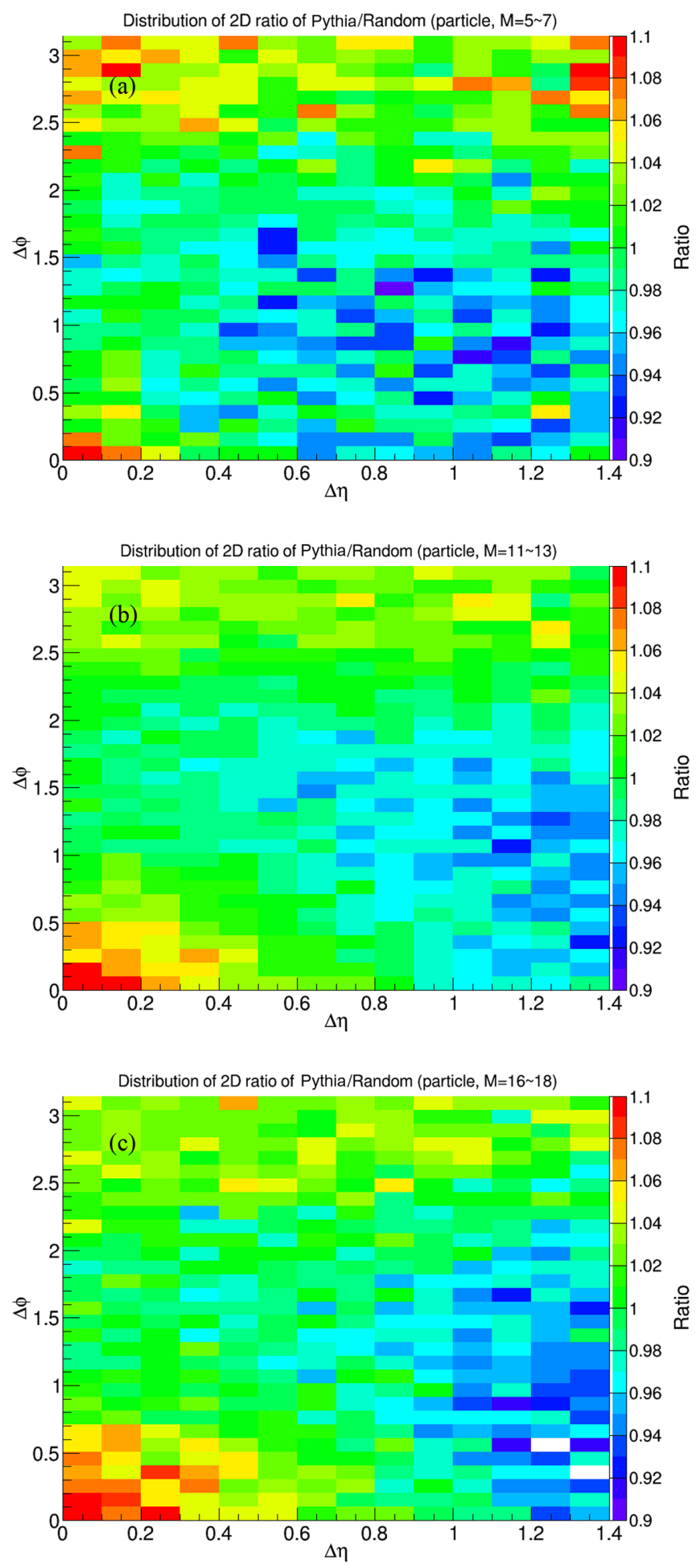

FIG. 14. 2D normalized two-particles correlation function $(d N / d \Delta \phi d \Delta \eta)_{(\text {PYTHIA })} /(d N / d \Delta \phi d \Delta \eta)_{(\text {Random })}$. The figures are produced by events with $M=11,12$, and 13 . The horizontal coordinate is $\Delta \eta$, and the vertical coordinate is $\Delta \phi$.

to the case of $M=5-7$ as shown in Fig. 13. We can understand the gross features of the cluster-cluster correlation in Fig. 15(b) in the following way. In the region of 
$\Delta \eta \sim 1.4$, the correlations for the random case is small, arising from the phase-space limitation of the triangular distribution of the particle-particle correlations as shown in 15(a). As $\Delta \eta$ decreases below the cluster radius $R$, those particles falling within the domain of the first cluster within a radius $R$ will become part of the other cluster, and thus, the probability of another cluster in the $(\Delta \eta, \Delta \phi)<R$ region of the first cluster is essentially zero when $K=2$ as indicated by a void in the $(\Delta \eta, \Delta \phi) \sim 0$ region. For the region at $(\Delta \eta \sim 0, \Delta \phi \sim \pi)$, there is a natural enhancement of the correlation because the relatively large value of the correlation function at $\Delta \eta \sim 0$ that enhances the formation of clusters at $\Delta \eta \sim 0$ and $\Delta \phi \sim \pi$.

Figure $15(\mathrm{~b})$ gives the 2D two-clusters correlation distribution of the $\Delta \phi$ and $\Delta \eta$ with $K=2$ obtained
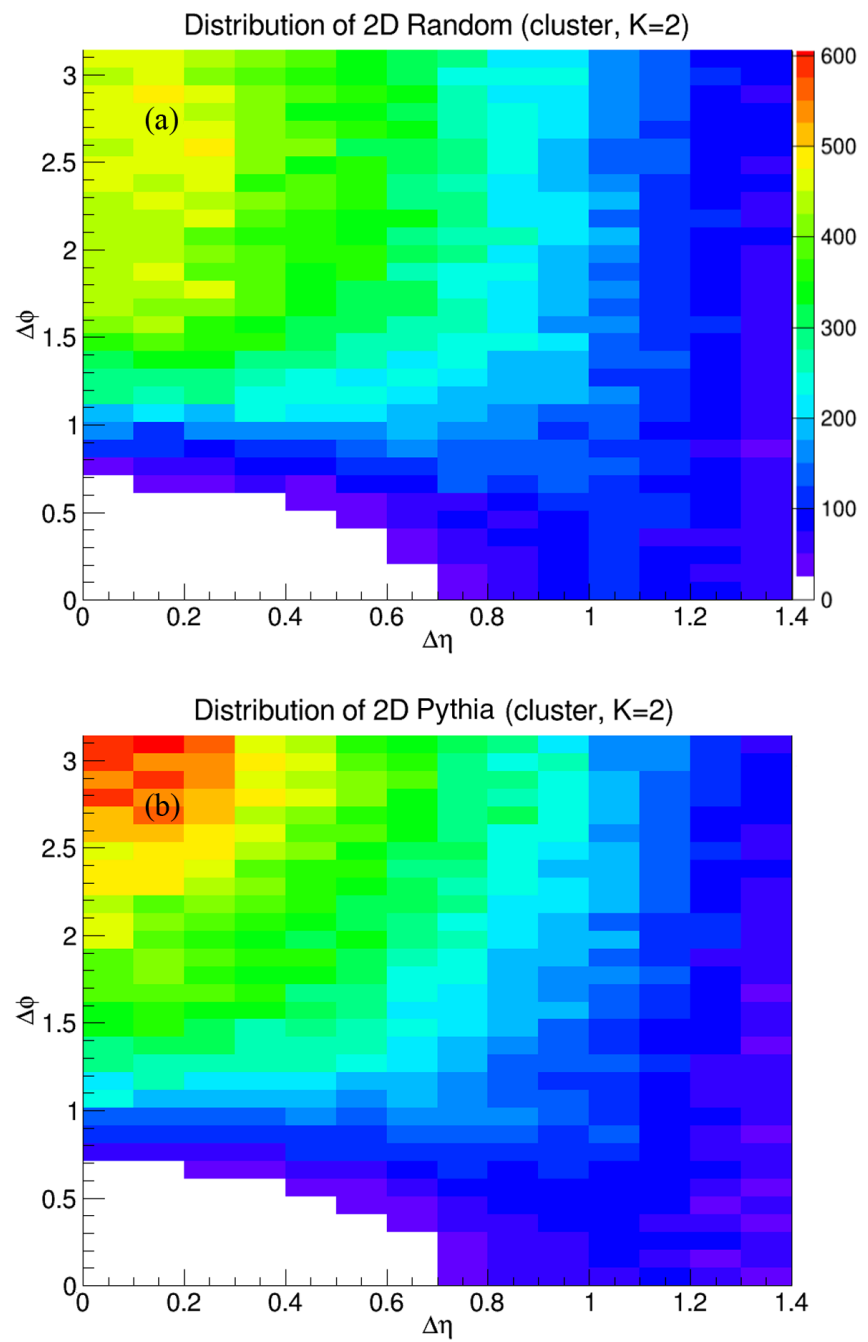

FIG. 15. Un-normalized 2D two-cluster correlation distribution $d N /(d \Delta \phi d \Delta \eta)$ is produced by events with two clusters. Figure (a) is the two-cluster $\Delta \eta-\Delta \phi$ correlation function for events from the random distribution, and Fig. (b) is the two-cluster $\Delta \eta-\Delta \phi$ correlation function for events generated by PYTHIA8.1. The $\Delta \phi$ and $\Delta \eta$ are positive as they are only differences of the azimuthal and pseudorapidity coordinates of the pair of clusters. with particles generated by PYTHIA8.1. This case of $K=2$ corresponds to the case of $M=5-7$ as shown in Fig. 13(b) and represents approximately the
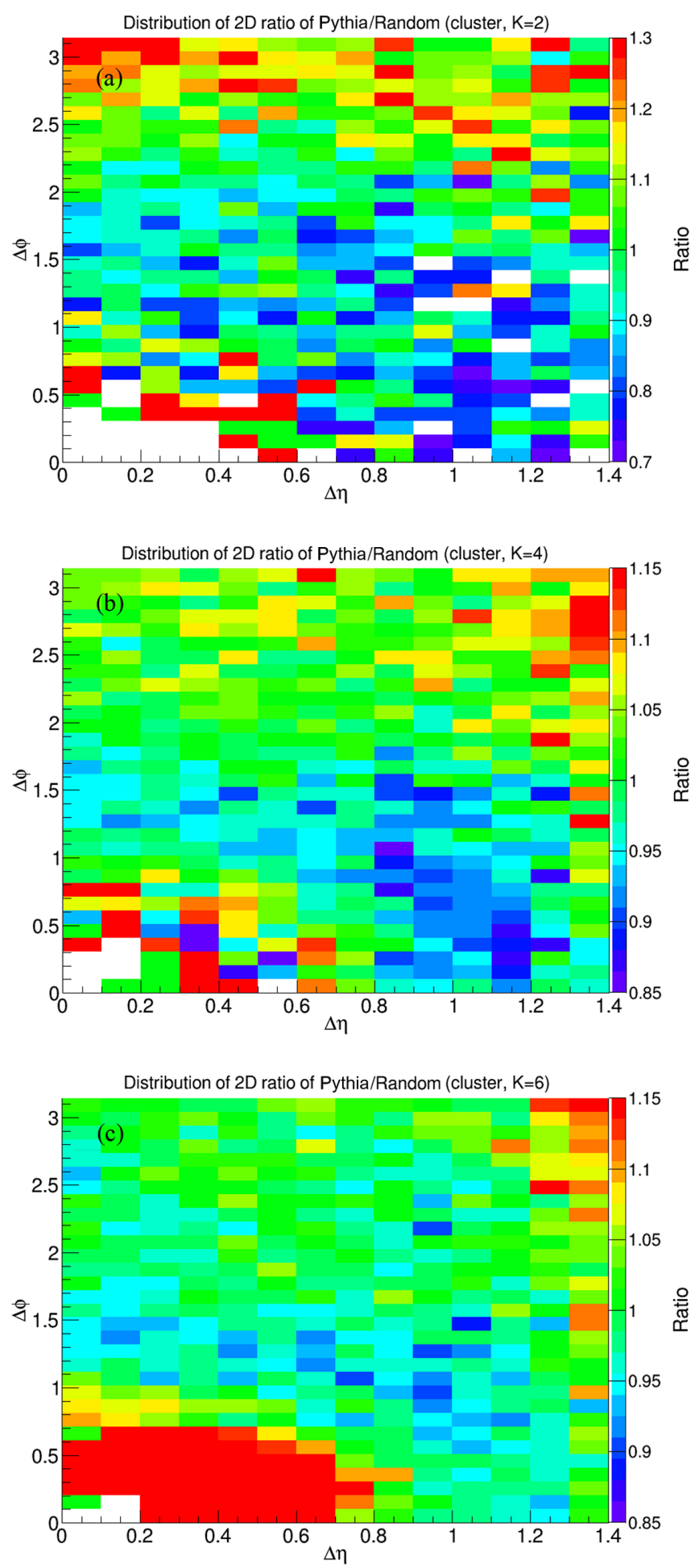

FIG. 16. Normalized 2D two-clusters correlation with function $(d N / d \Delta \phi d \Delta \eta)_{(\text {PYTHIA) }} /(d N / d \Delta \phi d \Delta \eta)_{\text {(Random) }}$. The figures are produced by events with 2,4 , and 6 clusters. The horizontal coordinate is the ratio of $\Delta \eta$, and the vertical coordinate is the ratio of $\Delta \phi$. 
minimum-biased case. The cluster-cluster correlation for the PYTHIA8.1 case retains some of the gross features as presented from the random case, arising from the phasespace limitation of the measurement windows. There is, however, finer differences arising from the dynamics of the PYTHIA8.1 particle generating processes. There appears to be an enhancement of the distribution at $\Delta \phi \sim \pi$ reflecting the occurrence of the back-to-back nature of the fragmentation processes as implemented in the PYTHIA8.1 program. The fine details show up more clearly upon taking the ratio $d N /\left.d \Delta \eta d \Delta \phi\right|_{\text {PYTHIA }} / d N /\left.d \Delta \eta d \Delta \phi\right|_{\text {random }}$ over the $(\Delta \eta, \Delta \phi)$ plane. Such a ratio will be called the normalized cluster-cluster correlation function for PYTHIA8.1 as show in Fig. 16(a).

The 2D normalized cluster-cluster correlation functions for $K=2,4$, and 6 in Fig. 16 exhibits the dynamics and its variation of the PYTHIA calculations as the cluster number changes. For $K=2$, the correlation shows a near-side jet in the region of $(\Delta \eta, \Delta \phi) \sim 0$. This near-side jet grows in strength as the cluster number $K$ increases. On the other hand, at $\Delta \phi \sim \pi$ there appears to be an away-side ridge along the $\eta$ direction for $K=2$. As $K$ increases, the ridge feature is modified to become a peak at $(\Delta \eta \sim 1.4, \Delta \phi \sim \pi)$ separated from the peak at $(\Delta \eta, \Delta \phi) \sim 0$.

It is instructive to compare the particle-particle corrections in Fig. 14 with their corresponding cluster-cluster correlations in Fig. 16. For the case of $M=5-7$ that corresponds closely with the case of $K=2$, one observes in both particle-particle and cluster-cluster correlations the occurrence of the near-side jet and the away-side ridge. The cluster-cluster correlation yields an amplified amplitude of about 30 percent, whereas the particle-particle correlation yields an amplitude of only about 10 percent. Thus, in comparison with the particle-particle correlation, the cluster-cluster correlation amplifies the amplitude for the away-side ridge to a greater degree. For the case of $M=10-13$ in comparison with $K=4$, the correlation at $(\Delta \eta, \Delta \phi) \sim 0$ is enhanced while the particle-particle correlation does not exhibit a large enhanced amplitude at that location; the cluster-cluster correlation exhibits an enhanced amplitude at $\Delta \eta \sim 1.4$ and $\Delta \phi \sim \pi$. For the case of $M=17-19$, or the corresponding case of $K=6$, the correlation at $(\Delta \eta, \Delta \phi) \sim 0$ is even more enhanced. Furthermore, the correlation function appears to be greatly enhanced at $(\Delta \eta \sim 1.4, \Delta \phi \sim \pi)$ indicating a regularity of the dynamics at a certain $\Delta \eta \sim 1.4$ in the awayside angles.

For completeness, there are additional pieces of information one can gain from the results of $d N / d \phi$ and $d N / d \eta$ distributions, which we shall present in the Appendix.

\section{CONCLUSIONS AND DISCUSSIONS}

The parton-parton hard scattering is an important process in high-energy nucleon-nucleon collisions. Although originally conceived to involve only the production of high- $p_{T}$ particles, it has been suggested that the dominance of the hard-scattering process may extend to the low- $p_{T}$ region with the production of minijets and mini-dijets as the collision energy increases.

As a first attempt to identify minijets, we develop an algorithm to search for clusters using the k-means clustering method, supplemented with a k-number (clusternumber) selection principle. The method adopts a scheme of random initialization of the initial centers, minimizing the potential function $\Phi(K)$ for a fixed $K$, and looking for the $K$ number of clusters that leaves the fewest number of particles $\Omega$ outside the cluster circles. The method is stable, fast, and yields clusters and their associated particles.

Using such a method, we have located clusters in the $(\eta, \phi)$ plane on an event-by-event basis, using events generated by PYTHIA8.1, which contains the dynamics of multiple parton interactions. To a cluster identified by the procedures, one often finds an associated cluster located at approximately $\left|\Delta \phi_{\text {jet-jet }}\right| \sim \pi \pm R$. Their azimuthal angular correlation suggests that they may be identified as the two partners of a mini-dijetlike pair. We find that clusters of low- $p_{T}$ hadrons are common occurrences for PYTHIA8.1 events with high multiplicities. The number of multiple clusters increases approximately linearly with increasing multiplicity $M$.

It must be pointed out, however, that clustering and azimuthal correlations alone cannot be the only means to identify minijets and mini-dijets. A randomly distributed set of particles in large multiplicities also exhibit clustering properties similar to those from the PYTHIA8.1 program with minijets. The ability to distinguish the dynamics of the particle production processes will require the measurement of the particle-particle and cluster-cluster correlations.

We have examined the particle-particle and clustercluster correlations obtained with particles generated from PYTHIA and compared these correlations with those from the random distribution. We need to normalize these correlations properly by dividing the correlation function obtained in PYTHIA by the correlation function obtained by a random distribution. We find that the normalized correlation function from PYTHIA has features that distinguish themselves from those of a random distribution. In this regard, the quantitative assessment of the dominance of the relativistic hard-scattering process in the low- $p_{T}$ region needs to be independently established in order to identify the clusters as physical minijets. The success of such an identification will provide a tool to investigate minijet and mini-dijet properties, for which not much detailed information has been collected. Furthermore, quantitative predictions based on first principles of perturbative QCD for the low- $p_{T}$ region is difficult because the multiple collision probability 
involves higher-order corrections beyond the leading order [72].

From our investigations, one may also wish to develop strategies to apply the proposed algorithm to examine experimental data at various energies and examine information on the production cross sections and the phasespace distribution of these objects, for comparison with the theory of multiple minijet production as a function of the collision energies. In this regard, we should note that the higher the $p p$ collision energy, the greater the probability is of the dominance of the hard-scattering process for the production of low- $p_{T}$ particles, and the greater the probability will be of the clusters to be physical minijets.

We have introduce a general method only as a first step towards our eventual goal of locating minijets. In future practical analysis in the search for minijets in experimental data, it may be reasonable to include a supplementary requirement that a minijet must contain at least a single particle with a $p_{T}$ greater than a certain threshold value $p_{0}$ (say $1 \mathrm{GeV} / \mathrm{c}$ ). Such a supplementary condition will serve the good purpose of fixing the minijet cluster number $K$ to facilitate the searching algorithm (in place of the present principle of the least number of outside points). It will reduce the number of clusters so that the effects of the finite size of the experimental windows is reduced. It will also bring us closer to the goal of examining minijets, multiple minijets, and their correlations. Future investigation along such directions, in conjunction with a properly modified k-means clustering algorithm, will be of great interest.

\section{ACKNOWLEDGMENTS}

The authors would like to thank Professors Zhenyu Ye and Soren Sorensen for helpful discussions. The research was supported in part by the Division of Nuclear Physics, U.S. Department of Energy under Contract No. DE-AC0500OR22725 with UT-Battelle and Contract No. DE-FG0288ER40424 with UCLA.

\section{APPENDIX A: THE ELBOW METHOD OF CLUSTER NUMBER SELECTION}

There is another method to select the cluster number $K$ by studying the $K$-dependence of the potential function $\Phi(K)$. For a given $K$ value, after the minimization of the potential function $\Phi(K)$ with respect to the random initialization of the cluster centers and the variations of the cluster center positions, the quantity $\Phi(K)$ of Eq. (3) is then evaluated. The potential function $\Phi(K)$ is, on the whole, a decreasing function of increasing $K$ (Fig. 17), as it reaches the limiting value of zero when the number of clusters $K$ is the same as the number of data points $M$. An inefficient and a slowly decreasing function of $\Phi(K)$ occurs, if a cluster is subdivided into smaller subclusters with a subsequently smaller change of the $\Phi(K)$ slope. On the other hand, a large and abrupt change of $\Phi(K)$ as a

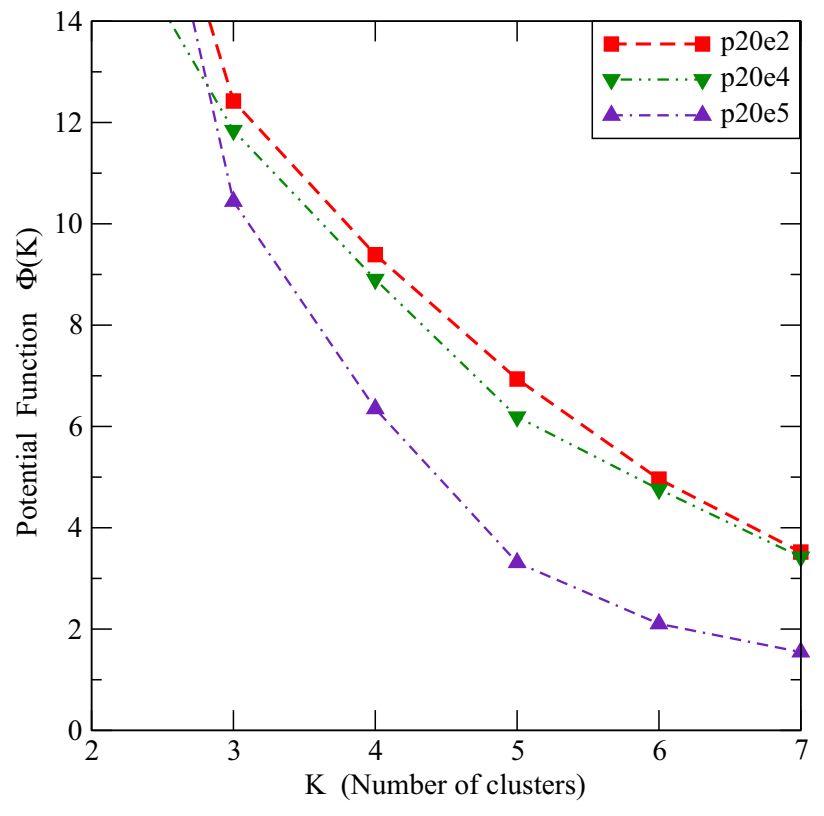

FIG. 17. Potential function $\Phi(K)$, the sum of square distances between the subset data points and their corresponding cluster centers, as a function of the number of clusters $K$, for minimumbias events with multiplicity $M=20$ generated by PYTHIA8.1.

function of $K$ signifies a significant change of the structure of the clustering configuration and may be the location of the appropriate cluster number. Hence, it has been suggested that the proper cluster number $K$ occurs at the kink (or elbow) of the curve of $\Phi(K)$ as a function of $K$ or at the location of an abrupt change of the slope of $\Phi(K)[85,90]$.

We calculate the potential function $\Phi(K)$ as a function of the cluster number $K$ for events with $M=20$ as shown in Fig. 4. For event p20e 2 shown in Fig. 4, a kink of $P(K)$ occurs at $K=3$, and a very weak kink also appears to occur at $K=6$. The determination of the location of the kink is not without ambiguity. The elbow method would suggest the cluster number of $K=3$ or 6 , but as we observed in Fig. 4, the proper cluster number as determined from the principle of fewest outside points is $K=7$. For event p20e4, kinks of $\Phi(K)$ occur at $K=3$ and 5, but the appropriate cluster number as determined from the principle of fewest outside points is 7. For p20e5, the potential function shows a sharp kink at $K=3$ and weaker kinks at 5 and 6, whereas the method of the principle of fewest outside points gives $K=6$. The method of the sharpest kink has the difficulty of recognizing the location of the kink, as many changes of slopes occur at different locations. If one takes the method to be given by the location with the greatest change of the magnitude of the slope, it would give $K$ numbers, which differ from the k-number selection principle of the fewest number of outside points.

We conclude that in the elbow method the determination on the location of the kink is ambiguous, and there is 
no obvious method to resolve the ambiguities. The principle of the fewest outside points should be the proper criterion for the selection of the proper cluster number $K$, as it is based on the physical property of the clustering of a minijet.

\section{APPENDIX B: TWO-PARTICLE AND TWO-CLUSTER $d N / d \Delta \eta$ AND $d N / d \Delta \phi$}

The results in Sec. VIII provide a wealth of information on the particle-particle and cluster-cluster correlation functions $d N / d \Delta \eta d \Delta \phi$ in the $(\Delta \eta, \Delta \phi)$ plane. The shape of the correlation function landscape contains many interesting features. In many measurements, it may be useful also to collect information on the "marginal distributions" $d N / d \Delta \eta$ and $d N / d \Delta \phi$ by integrating the twodimensional distribution $d N / d \Delta \eta d \Delta \phi$ over $\Delta \eta$ or $\Delta \phi$ directions as

$$
\begin{aligned}
\frac{d N}{d \Delta \eta} & =\sum_{\Delta \phi} \frac{d N}{d \Delta \eta d \Delta \phi} d \Delta \phi, \\
\text { and } \frac{d N}{d \Delta \phi} & =\sum_{\Delta \eta} \frac{d N}{d \Delta \eta d \Delta \phi} d \Delta \eta .
\end{aligned}
$$

These marginal distributions are not as informative as the full two-dimensional $d N / d \Delta \eta d \Delta \phi$ distribution because many important features may become obscured when the two dimensional distribution has been integrated. They provide partial information on the particle or cluster distribution projected in certain directions. Recognizing that these marginal distributions are only part of the full distribution, we shall show here the marginal distributions for various values of particle numbers $M$ and cluster number $K$, for completeness.

\section{Two-particle $d N / d \Delta \eta$ and $d N / d \Delta \phi$}

Fig. 18 gives the un-normalized two-particle correlation distribution with $M \sim 5-7$. The quantity $d N / d \Delta \phi$ in Fig. 18(a) shows a nearly flat distribution for the random distribution but a back-to-back correlation for the PYTHIA8.1 calculations. The quantity $d N / d \Delta \eta$ in Fig. 18(b) shows a triangular distribution because of the $\eta$ window. The difference between the distributions from PYTHIA8.1 and from the random distribution is small.

To illustrate the finer differences between the distributions, we defined normalized distributions as

$$
\text { normalized } \frac{d N}{d \Delta \eta}=\frac{\frac{d N}{\left.d \Delta \eta\right|_{\text {PYTHIA }}}}{\frac{d N}{\left.d \Delta \eta\right|_{\text {random }}}}
$$

and
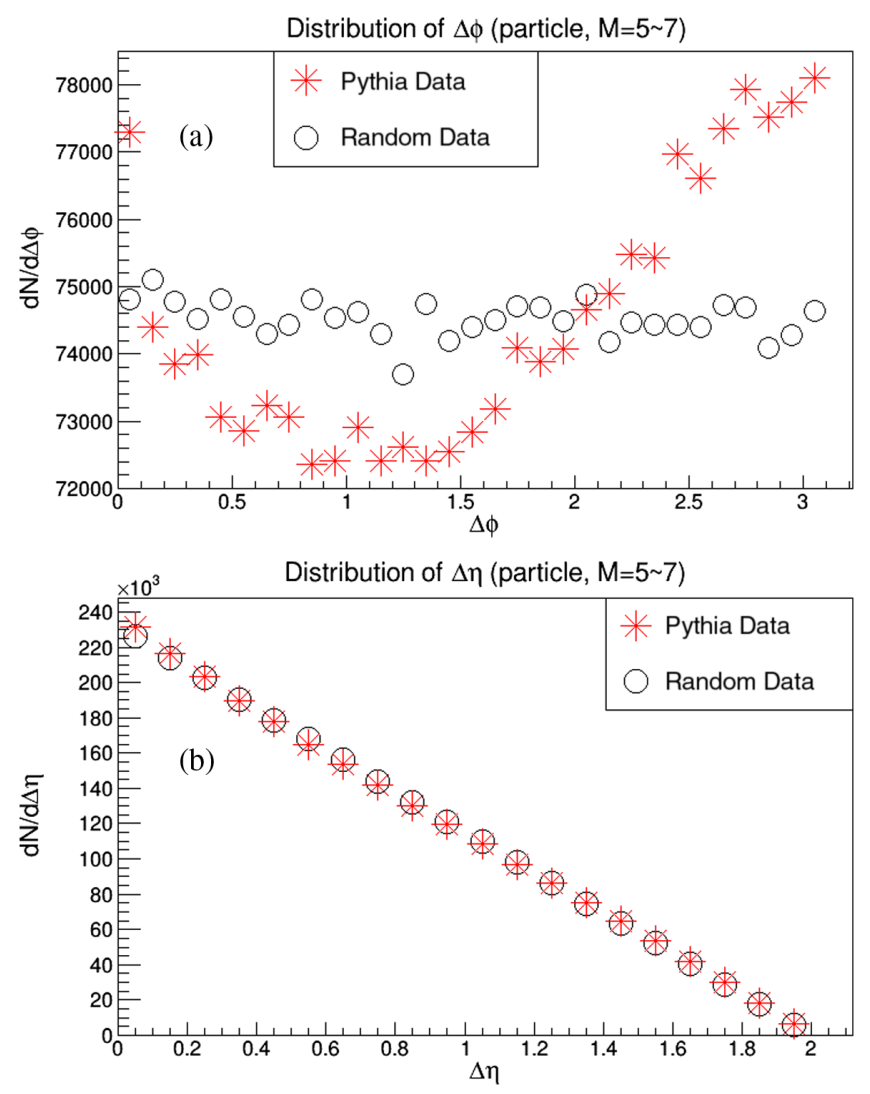

FIG. 18. Un-normalized two-particle differential correlation distribution (a) $d N / d \Delta \phi$ and (b) $d N / d \Delta \eta$, for events with multiplicity $M=5 \sim 7$. The red inverted triangle points are for events generated by PYTHIA8.1, and the black circular points are from events generated from a random distribution.

$$
\text { normalized } \frac{d N}{d \Delta \phi}=\frac{\frac{d N}{\left.d \Delta \phi\right|_{\text {PYTHI }}}}{\frac{d N}{\left.d \Delta \phi\right|_{\text {random }}}} .
$$

We display the normalized distribution for $\Delta \phi$ and $\Delta \eta$ for $M \sim 5-7$ in Fig. 19(a) and (b) and for $M \sim 11-13$ in Fig. 19(c) and (d). The minijet component shows up as a peak at $\Delta \phi \sim 0$ in $d N / d \Delta \phi$ in Fig. 19(a) and (c). The away-side back-to-back correlation appears as a rising peak of $d N / d \Delta \phi$ at $\Delta \phi \sim \pi$ in Fig. 19(a) and (c). The near-side jet shows up as peak at $\eta \sim 0$ in $d N / d \Delta \eta$ and shows up at both $M \sim 5-7$ in Fig. 19(b) and (d). The $d N / d \eta$ at large $\Delta \eta$ gives a peak at large $\eta$ for $M \sim 5-7$, but the distribution decreases for large values of $M$ in Fig. 19(d). The away-side peak has a smaller magnitude as $K$ increases, expected by the dilution effect of momentum conservation.

\section{Two-cluster $d N / d \Delta \eta$ and $d N / d \Delta \phi$}

Figure 20(a) and (b) give the un-normalized two-clusters correlation distribution $d N / d \Delta \phi$ and $d N / d \Delta \eta$, respectively, for $K=2$. The $d N / d \Delta \phi$ distribution is nearly flat for $\Delta \phi \sim \pi$ but suppressed near the region near $\Delta \phi \sim 0$ 

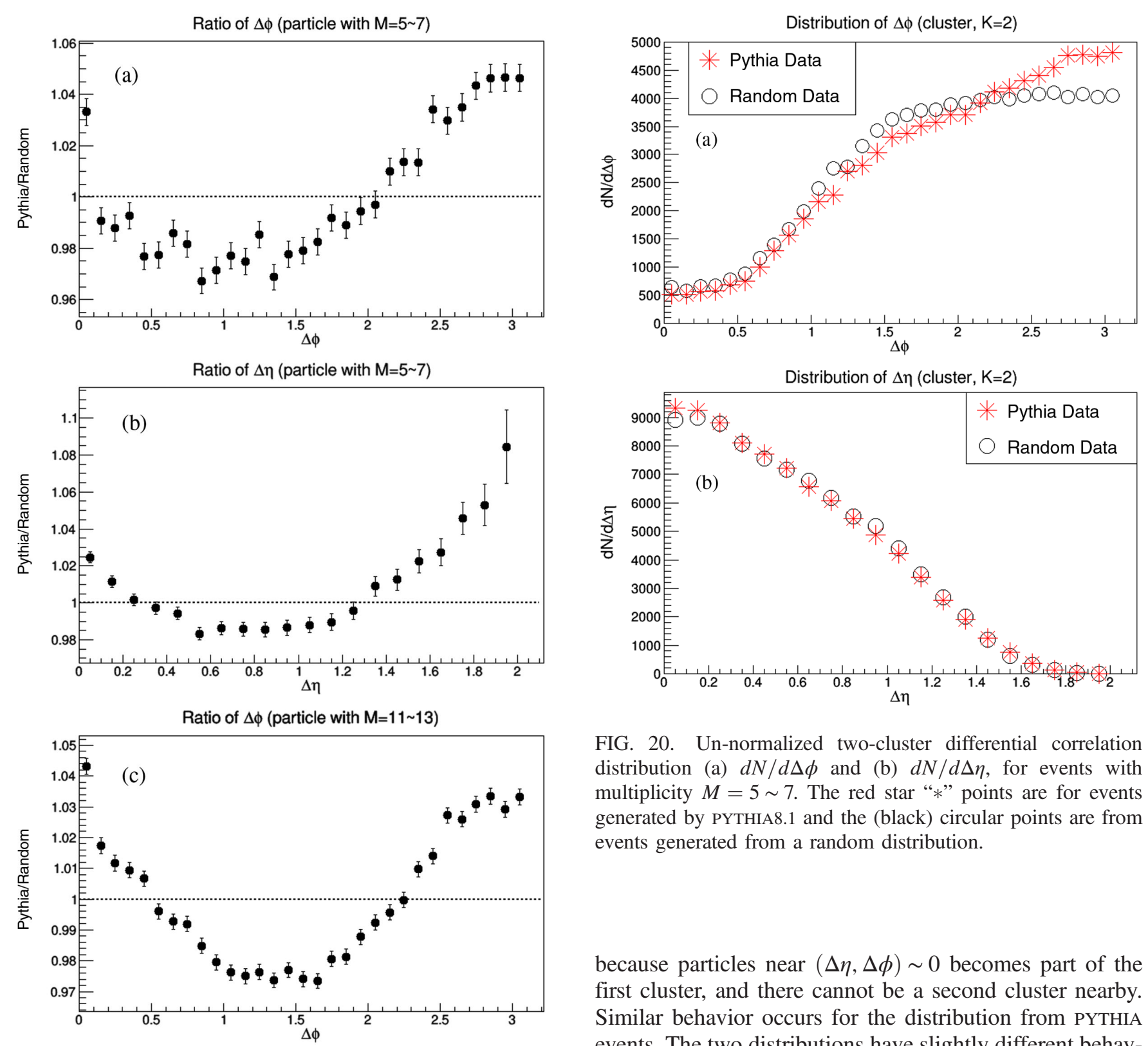

FIG. 20. Un-normalized two-cluster differential correlation distribution (a) $d N / d \Delta \phi$ and (b) $d N / d \Delta \eta$, for events with multiplicity $M=5 \sim 7$. The red star " * " points are for events generated by PYTHIA8.1 and the (black) circular points are from events generated from a random distribution.

because particles near $(\Delta \eta, \Delta \phi) \sim 0$ becomes part of the first cluster, and there cannot be a second cluster nearby. Similar behavior occurs for the distribution from PYTHIA events. The two distributions have slightly different behaviors at $\Delta \phi \sim \pi$, arising from the occurrence of back-to-back correlations in dijet events. The distribution in Fig. 20(b) for $d N / d \Delta \eta$ is similar to the triangular distribution in Fig. 18(b), except that the distribution decreases faster to zero at a smaller value of $\Delta \eta \sim 1.6$ instead of $\Delta \eta \sim 2.0$ in Fig. 18(b).

We show the normalized $d N / d \Delta \phi$ and $d N / d \Delta \eta$ for $K=2$ in Fig. 21(a) and Fig. 21(b), for $K=4$ in Fig. 21(c) and Fig. 21(d), and for $K=6$ in Fig. 21(e) and Fig. 21(f). While these distributions collaborate what one can find out about the shape of the distributions in the full twodimensional $d N / d \Delta \eta d \Delta \phi$ distribution, it is difficult to extract information on the two-dimensional distribution from the marginal distributions. What can be stated is that as a function of increasing $K$ values, the normalized $d N / d \Delta \phi$ distribution has a peak at $\Delta \phi \sim \pi$ that remains for $K=4$ and 6 . 

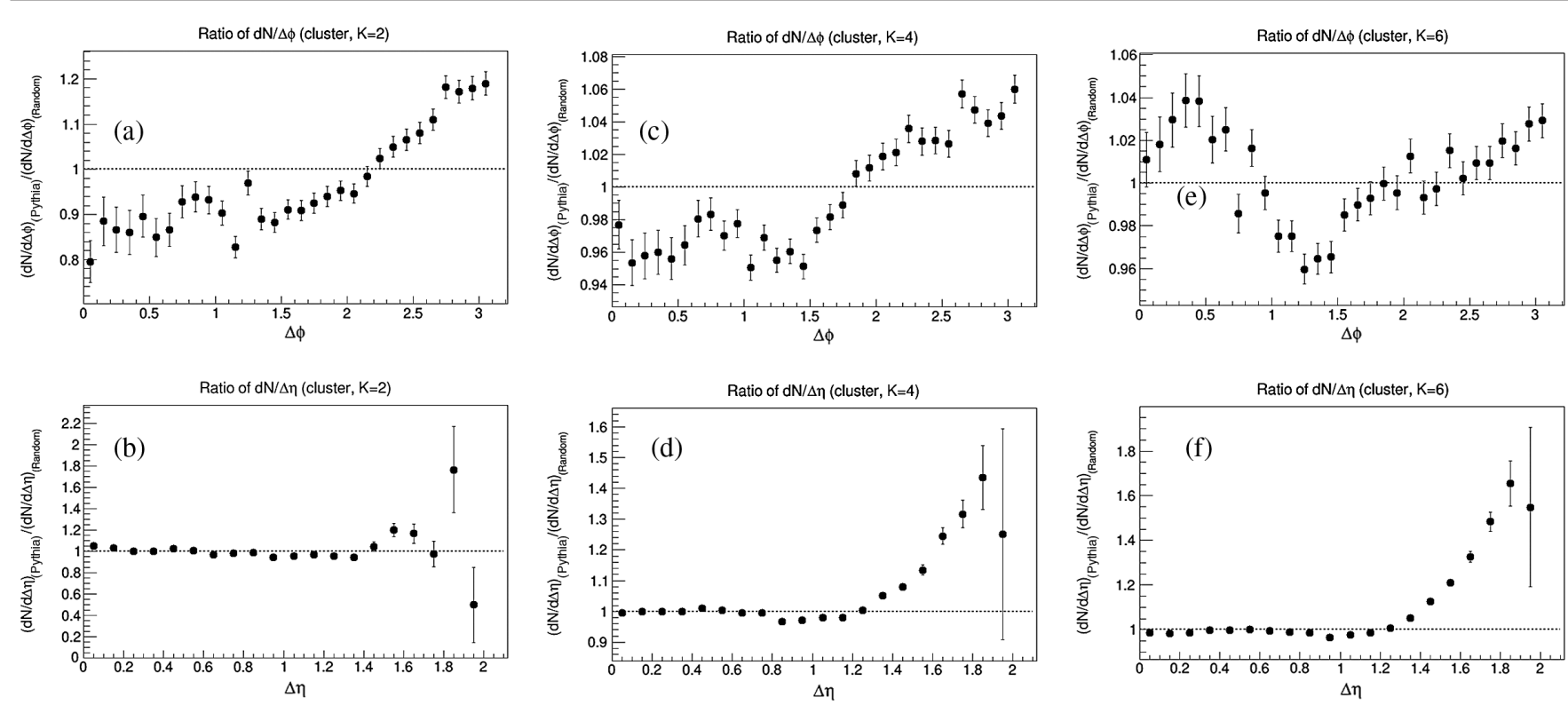

FIG. 21. Normalized two-clusters correlation distribution. The figures are produced by PYTHIA8.1, generated and random events with 2,4 , and 6 clusters. The Figs. (a), (c), and (e) are the ratio of $\Delta \phi$ with function $(d N / d \Delta \phi)_{(\text {PYTHIA })} /(d N / d \Delta \phi)_{(\text {Random })}$, and the Figs. (b), (d), and (f) are the ratio of $\Delta \eta$ with function $(d N / d \Delta \eta)_{\text {(PYTHIA) }} /(d N / d \Delta \eta)_{\text {(Random) }}$.

[1] R. Blankenbecler and S. J. Brodsky, Phys. Rev. D 10, 2973 (1974); R. Blankenbecler, S. J. Brodsky, and J. Gunion, Phys. Rev. D 12, 3469 (1975); E. A. Schmidt and R. Blankenbecler, Phys. Rev. D 15, 3321 (1977); R. Blankenbecler, A. Capella, J. Tran Thanh Van, C. Pajares, and A. V. Ramallo, Phys. Lett. 107B, 106 (1981).

[2] A. L. S. Angelis et al. (CCOR Collaboration), Phys. Lett. 79B, 505 (1978).

[3] R. P. Feynman, R. D. Field, and G. C. Fox, Phys. Rev. D 18, 3320 (1978).

[4] J. F. Owens, E. Reya, and M. Glück, Phys. Rev. D 18, 1501 (1978); D. W. Duke and J. F. Owens, Phys. Rev. D 30, 49 (1984).

[5] J. Rak and M. J. Tannenbaum, High- p $_{T}$ Physics in the Heavy Ion Era (Cambridge University Press, Cambridge, England, 2013).

[6] T. Sjöstrand, Comput. Phys. Commun. 39, 347 (1986); T. Sjöstrand and M. Bengtsson, Comput. Phys. Commun. 43, 367 (1987).

[7] T.Sjöstrand and M. van Zijl, Phys. Rev. D 36, 2019 (1987).

[8] T. Sjöstrand, S. Mrenna, and P. Skands, J. High Energy Phys. 05 (2006) 026.

[9] T. Sjöstrand, S. Mrenna, and P. Skands, Comput. Phys. Commun. 178, 852 (2008).

[10] T. Sjöstrand, S. Ask, J. R. Christiansen, R. Corke, N. Desai, P. Ilten, S. Mrenna, S. Prestel, C. O. Rasmussen, and P. Z. Skands, Comput. Phys. Commun. 191, 159 (2015).

[11] T. Sjöstrand and P.Z. Skands, J. High Energy Phys. 03 (2005) 053.
[12] T. Sjöstrand and P.Z. Skands, Eur. Phys. J. C 39, 129 (2003).

[13] R. Corke and T. Sjöstrand, J. High Energy Phys. 03 (2011) 032.

[14] R. Corke and T. Sjöstrand, J. High Energy Phys. 01 (2010) 035.

[15] C. Albajar et al. (UA1 Collaboration), Nucl. Phys. B309, 405 (1988).

[16] K. S. Eskola, K. Kajantie, and J. Lindfors, Nucl. Phys. B323, 37 (1989).

[17] X. N. Wang and M. Gyulassy, Phys. Rev. D 44, 3501.

[18] G. Calucci and D. Treleani, Phys. Rev. D 41, 3367 (1990).

[19] G. Calucci and D. Treleani, Phys. Rev. D 44, 2746 (1991).

[20] G. Calucci and D. Treleani, Int. J. Mod. Phys. A 06, 4375 (1991).

[21] G. Calucci and D. Treleani, Phys. Rev. D 49, 138 (1994).

[22] G. Calucci and D. Treleani, Phys. Rev. D 50, 4703 (1994).

[23] G. Calucci and D. Treleani, Phys. Rev. D 63, 116002 (2001).

[24] A. Accardi and D. Treleani, Phys. Rev. D 64, 116004 (2001).

[25] C. Y. Wong, Introduction to High-Energy Heavy-Ion Collisions (World Scientific, Singapore, 1994).

[26] C. Y. Wong and G. Wilk, Acta Phys. Pol. B 43, 2047 (2012).

[27] C. Y. Wong and G. Wilk, Phys. Rev. D 87, 114007 (2013).

[28] C. Y. Wong, G. Wilk, L. J. L. Cirto, and C. Tsallis, EPJ Web Conf. 90, 04002 (2015).

[29] C. Y. Wong, G. Wilk, L. J. L. Cirto, and C. Tsallis, Phys. Rev. D 91, 114027 (2015).

[30] Y. Nambu, Lectures at Copenhagen Symposium (1970). 
[31] J. D. Bjorken, Proceedings of the Summer Institute on Particle Physics, edited by Zipt, SLAC-167 (1973).

[32] A. Casher, J. Kogut, and L. Susskind, Phys. Rev. D 10, 732 (1974); A. Casher, H. Neuberger, and S. Nussinov, Phys. Rev. D 20, 179 (1979); 21, 1966 (1980).

[33] J. Schwinger, Phys. Rev. 128, 2425 (1962); in Theoretical Physics, Trieste Lectures, 1962 (I.A.E.A., Vienna, 1963), p. 89.

[34] X. Artru and G. Mennessier, Nucl. Phys. B70, 93 (1974).

[35] B. Andersson, G. Gustafson, and C. Peterson, Z. Phys. C 1, 105 (1979); B. Andersson, G. Gustafson, and B. Söderberg, Z. Phys. C 20, 317 (1983).

[36] A comprehensive review of the application of the flux tube fragmentation model for nucleon-nucleon and $e^{+}-e^{-}$ collisions can be found in B. Andersson, G. Gustafson, G. Ingelman, and T. Sjöstrand, Phys. Rep. 97, 31 (1983); X. Artru, Phys. Rep. 97, 147 (1983).

[37] X. Artru, Z. Phys. C 26, 83 (1984).

[38] B. Andersson, G. Gustafson, and T. Sjöstrand, Z. Phys. C 20, 317 (1983); T. Sjöstrand and M. Bengtsson, Comput. Phys. Commun. 43, 367 (1987); B. Andersson, G. Gustavson, and B. Nilsson-Alqvist, Nucl. Phys. B281, 289 (1987).

[39] J. Schwinger, Phys. Rev. 82, 664 (1951).

[40] R. C. Wang and C. Y. Wong, Phys. Rev. D 38, 348 (1988).

[41] H-P. Pavel and D. Brink, Z. Phys. C 51, 119 (1991).

[42] C. Y. Wong, R. C. Wang, and C. C. Shih, Phys. Rev. D 44, 257 (1991).

[43] C. Y. Wong and R. C. Wang, Phys. Rev. D 44, 679 (1991).

[44] G. Gatoff and C. Y. Wong, Phys. Rev. D 46, 997 (1992); C. Y. Wong and G. Gatoff, Phys. Rep. 242, 489, 489 (1994).

[45] C. Y. Wong, R. C Wang, and J. S. Wu, Phys. Rev. D 51, 3940 (1995).

[46] D. A. Derkach and G. A. Feofilov, Phys. At. Nucl. 71, 2087 (2008); E. O. Bodnya, V. N. Kovalenko, A. M. Puchkov, and G. A. Feofilov, AIP Conf. Proc. 1606, 273 (2014); J. Adam et al. J. High Energy Phys. 05 (2015) 097; G. Feofilov, I. Altsybeev, and O. Kochebina, Proc. Sci., ISHEPP2014 (2014) 067.

[47] L. McLerran and R. Venugopalan, Phys. Rev. D 50, 2225 (1994); J. Jalilian-Marian, A. Kovner, L. McLerran, and H. Weigert, Phys. Rev. D 55, 5414 (1997).

[48] L. McLerran, Lect. Notes Phys. 583, 291 (2002).

[49] L. McLerran, Prog. Theor. Phys. Suppl. 187, 17 (2011).

[50] L. McLerran, Acta Phys. Pol. B 41, 2799 (2010).

[51] A. Kovner, L. McLerran, and H. Weigert, Phys. Rev. D 52 , 3809 (1995); 52, 6231 (1995).

[52] F. Geilis, T. Lappi, and L. McLerran, Nucl. Phys. A828, 149 (2009); K. Fukushima, F. Gelis, and T. Lappi, Nucl. Phys. A831, 184 (2009); F. Gelis and N. Tanji, arxiv:1510.05454.

[53] C. Y. Wong and R. Blankenbecler, Phys. Rev. C 23, 2433 (1978).

[54] The hard-scattering jet-production invariant cross section at mid-rapidity varies approximately as $\alpha_{s}^{2} \sqrt{\sqrt{s} / p_{T}} / p_{T}^{4}$, as a function of the $p p$ collision energy $\sqrt{s}$ and the jet $p_{T}$ at high collision energies, (see for example the analytical expression of Eq. (14) of [29], obtained by integrating approximate parton structure functions).

[55] J. Adams et al., Phys. Rev. Lett. 95, 152301 (2005).

[56] J. Adams et al. (STAR Collaboration), Phys. Rev. C 74, 032006 (2006).
[57] R. J. Porter and T. A. Trainor (STAR Collaboration), J. Phys. Conf. Ser. 27, 98 (2005).

[58] T. A. Trainor and R. L. Ray, Phys. Rev. C 84, 034906 (2011).

[59] J. Adams et al. (STAR Collaboration), Phys. Rev. Lett. 95, 152301 (2005).

[60] J. Adams et al. (STAR Collaboration), Phys. Rev. C 73, 064907 (2006).

[61] J. Putschke (STAR Collaboration), J. Phys. G 34, S679 (2007).

[62] J. Bielcikova (STAR Collaboration), J. Phys. G 34, S929 (2007).

[63] F. Wang (STAR Collaboration), Int. J. Mod. Phys. E 16, 3168 (2008).

[64] C. Y. Wong, Phys. Rev. C 76, 054908 (2007).

[65] C. Y. Wong, Phys. Rev. C 78, 064905 (2008).

[66] C. Y. Wong, Phys. Rev. C 80, 034908 (2009).

[67] A. Adare et al. (PHENIX Collaboration), Phys. Rev. C 78, 014901 (2008); Phys. Rev. D 83, 052004 (2011); Phys. Rev. C 83, 064903 (2011).

[68] T. Akesson et al. (Axial Field Spectrometer Collaboration), Z. Phys. C 34, 163 (1987).

[69] F. Abe et al. (CDF Collaboration), Phys. Rev. Lett. 79, 584 (1997); Phys. Rev. D 56, 3811 (1997).

[70] V. M. Abazov et al. (D0 Collaboration), Phys. Rev. D 81, 052012 (2010).

[71] S. Chatrchyan et al. (CMS Collaboration), Phys. Rev. D 89, 092010 (2014).

[72] P. Kotko, A. M. Stasto, and M. Strikman, Phys. Rev. D 95, 054009 (2017).

[73] V. Khachatryan et al. (CMS Collaboration), Phys. Rev. Lett. 116, 172302 (2016).

[74] V. Khachatryan et al. (CMS Collaboration), Phys. Lett. B 765, 193 (2017).

[75] A. Milov (ATLAS Collaboration), Nucl. Part. Phys. Proc. 289-290, 470 (2017).

[76] K. Burka (ATLAS Collaboration), Proc. Sci., DIS2016 (2016) 053.

[77] V. Zaccolo (ALICE Collaboration), Charged-particle multiplicity distributions over wide pseudorapidity range in proton-proton and proton-lead collisions with ALICE, CERN Report No. CERN-THESIS-2015-364, 2015.

[78] E. Pereira De Oliveira Filho (ALICE Collaboration), Study of the angular correlation between heavy-flavour decay electrons and charged unidentified particles in pp and $\mathrm{p}-\mathrm{Pb}$ collisions with ALICE, CERN Report No. CERN-THESIS2014-373, 2014.

[79] C. Y. Wong, Phys. Rev. C 84, 024901 (2011).

[80] CMS Collaboration, J. High Energy Phys. 09 (2010) 091.

[81] C. Y. Wong, J. Phys. G 44, 075102 (2017).

[82] D. M. Kaplan et al., Phys. Rev. Lett. 40, 435 (1978).

[83] C. Y. Wong and H. Wang, Phys. Rev. C 58, 376 (1998).

[84] D. W. Duke and J. F. Owens, Phys. Rev. D 30, 49 (1984); J. F. Owens, Rev. Mod. Phys. 59, 465 (1987).

[85] For an online introduction to k-means method, see J. Ng, Lectures in Machine Learning (Stanford University, 2017), https://www.coursera.org/learn/machine-learning/lecture/ czmip/unsupervised-learning-introduction.

[86] H. Steinhaus, Bull. Acad. Pol. Sci., Sér. sci. tech. 4, 801 (1957) (in French).

[87] S. P. Lloyd, IEEE Trans. Inf. Theory 28, 129 (1982). 
[88] J. B. MacQueen, Some methods for classification and analysis of multivariate observations, in Proceedings of 5th Berkeley Symposium on Mathematical Statistics and Probability (University of California Press, Berkeley, CA, USA, 1967), pp. 281-297.

[89] D. Arthur and S. Vassilvitskii, Proceedings of the Eighteenth Annual ACM-SIAM Symposium on Discreet
Algorithm, Society for Industrial and Applied Mathematics, Philadelphia, PA, USA (2007), p. 1027.

[90] R. L. Thorndike, Psychometrika 18, 267 (1953).

[91] S. Chekanov, Eur. Phys. J. C 47, 611 (2006).

[92] CMS Collaboration, J. High Energy Phys. 07 (2011) 076.

[93] V. Khachatryan et al. (CMS Collaboration), J. High Energy Phys. 09 (2010) 091; 07 (2011) 076. 